\title{
$\Omega$-deformation of B-twisted gauge theories and the 3d-3d correspondence
}

\author{
Yuan Luo, ${ }^{a}$ Meng-Chwan Tan, ${ }^{a}$ Junya Yagi ${ }^{b, c}$ and Qin Zhao ${ }^{a}$ \\ ${ }^{a}$ Department of Physics, National University of Singapore, \\ 2 Science Drive 3, 117551 Singapore \\ ${ }^{b}$ International School for Advanced Studies (SISSA), \\ via Bonomea 265, 34136 Trieste, Italy \\ ${ }^{c}$ INFN, Sezione di Trieste, \\ via Valerio 2, 34149 Trieste, Italy \\ E-mail: yuanluo@nus.edu.sg, mctan@nus.edu.sg, junya.yagi@sissa.it, \\ zhaoqin@u.nus.edu
}

ABSTRACT: We study $\Omega$-deformation of B-twisted gauge theories in two dimensions. As an application, we construct an $\Omega$-deformed, topologically twisted five-dimensional maximally supersymmetric Yang-Mills theory on the product of a Riemann surface $\Sigma$ and a three-manifold $M$, and show that when $\Sigma$ is a disk, this theory is equivalent to analytically continued Chern-Simons theory on $M$. Based on these results, we establish a correspondence between three-dimensional $\mathcal{N}=2$ superconformal theories and analytically continued Chern-Simons theory. Furthermore, we argue that there is a mirror symmetry between $\Omega$-deformed two-dimensional theories.

Keywords: Supersymmetric gauge theory, Chern-Simons Theories, Field Theories in Higher Dimensions, M-Theory

ARXIV EPRINT: 1410.1538

In memory of Tan See Hong. 


\section{Contents}

1 Introduction 1

$2 \quad \Omega$-deformation of B-twisted gauge theories $\quad 4$

2.1 Supersymmetry transformation laws 4

$\begin{array}{lll}2.2 & \text { Supersymmetric action } & 6\end{array}$

2.3 Boundary condition $\quad 8$

$\begin{array}{llr}2.4 \text { Localization } & 10\end{array}$

3 Chern-Simons theory from 5d MSYM theory 13

$\begin{array}{lll}3.1 \Omega \text {-deformed twisted 5d MSYM theory on } \Sigma \times M & 13\end{array}$

$\begin{array}{lll}3.2 & \text { Localization to analytically continued Chern-Simons theory } & 16\end{array}$

4 3d-3d correspondence $\quad 19$

4.1 $T[M]$ and analytically continued Chern-Simons theory 20

$\begin{array}{lll}4.2 \Omega \text {-deformed mirror symmetry } & 21\end{array}$

\section{Introduction}

The primary motivation for this work is to better understand the $3 \mathrm{~d}-3 \mathrm{~d}$ correspondence via five-dimensional maximally supersymmetric Yang-Mills (5d MSYM) theory. In trying to do so, we are naturally led to study $\Omega$-deformation of B-twisted gauge theories in two dimensions, which is another theme of the present paper.

The 3d-3d correspondence associates to every three-manifold $M$ an $\mathcal{N}=2$ superconformal field theory $T[M]$ in three dimensions. (Early works on the subject are [1-7].) A key fact about $T[M]$ is that it is closely related to Chern-Simons theory on $M$ with complex gauge group. For instance, the partition functions of $T[M]$ on $S^{1} \times S^{2}$ and the squashed three-sphere $S_{b}^{3}$ are equal to those of complex Chern-Simons theory at level $k=0[8,9]$ and $k=1$ [10], respectively. More generally, it has been proposed recently [11] that the partition function of $T[M]$ on the squashed lens space $L(k, 1)_{b}$ equals that of complex Chern-Simons theory at level $k$.

We are interested in a variant of these relations where $T[M]$ on $S^{1} \times_{\varepsilon} D$ is equated to analytically continued Chern-Simons theory [12-14], which is the holomorphic part of complex Chern-Simons theory. Here $S^{1} \times_{\varepsilon} D$ is a twisted product of $S^{1}$ and a disk $D$, with parameter $\varepsilon$. This version is actually more powerful, in the sense that the partition functions on $S^{1} \times_{\varepsilon} D$ with various boundary conditions give holomorphic blocks of the theory $[15,16]$, and the partition function on $L(k, 1)_{b}$ factorizes into these blocks and their 
complex conjugates. ${ }^{1}$ A derivation of this version was provided by Beem et al. [16], whose argument built on earlier work of Witten [20,21].

One of the main results of this paper is an alternative derivation of this last relation. More precisely, we establish the equivalence between the $Q$-invariant sector of $T[M]$ on $S^{1} \times_{\varepsilon} D$ and analytically continued Chern-Simons theory on $M$, where $Q$ is a certain supercharge.

The said equivalence is an example of various correspondences between theories in $d$ and $6-d$ dimensions that originate from the $\mathcal{N}=(2,0)$ superconformal theory in six dimensions. The best-known among these is probably the AGT correspondence $[22,23]$ relating $4 \mathrm{~d} \mathcal{N}=2$ theories and Toda theory, which one obtains by considering the $(2,0)$ theory compactified and topologically twisted on Riemann surfaces. In our case, the correspondence originates from the $(2,0)$ theory formulated on $S^{1} \times{ }_{\varepsilon} D \times M$, with topological twisting along the three-manifold $M$. The general idea is the following. When $M$ is very small, this theory reduces to $T[M]$ on $S^{1} \times_{\varepsilon} D$. On the other hand, if one somehow integrates out the degrees of freedom propagating along $S^{1} \times{ }_{\varepsilon} D$, one should get a theory on $M$. The $Q$-invariant sector of the latter is, presumably, analytically continued Chern-Simons theory. The correspondence in question then follows by identifying the two theories coming from the same $6 \mathrm{~d}$ theory.

Although the idea may be clear, showing that we indeed get analytically continued Chern-Simons theory is difficult if we stay within six dimensions, since the $(2,0)$ theory has no known Lagrangian description. To avoid this difficulty, we consider the limit where the radius $R$ of the $S^{1}$ is very small. This allows us to describe the $6 \mathrm{~d}$ theory as $5 \mathrm{~d}$ MSYM theory on $D \times M$, and write down the Lagrangian explicitly. Then we can apply localization techniques to simplify the path integral for correlation functions of $Q$-invariant operators. We will show that the path integral for the $5 \mathrm{~d}$ theory is equivalent to that for analytically continued Chern-Simons theory, and explain how this result can be used to establish the claimed equivalence for finite $R$. The logic of our argument is essentially the same as those employed in [8,9] for the $S^{1} \times S^{2}$ case or [10] for the $S_{b}^{3}$ case. (A similar approach was taken in $[24,25]$ to establish the equivalence between a twisted 5d MSYM theory compactified on $S^{3}$ and $q$-deformed Yang-Mills theory in two dimensions.)

The construction of the $5 \mathrm{~d}$ theory is, however, nontrivial and interesting on its own, and this takes us to the second theme of the present work. That is the $\Omega$-deformation of B-twisted gauge theories.

The nontriviality comes from the fact that we are reducing the $6 \mathrm{~d}$ theory on the nontrivial $D$-fibration $S^{1} \times_{\varepsilon} D$ over $S^{1}$, constructed by gluing the fiber with a rotation by angle $2 \pi R \varepsilon$. This rotation induces a deformation of the resulting $5 \mathrm{~d}$ MSYM theory on $D \times M$. To understand what kind of deformation is induced, suppose we further dimensionally reduce the $5 \mathrm{~d}$ theory on $M$; thus, in total, we are reducing the $6 \mathrm{~d}$ theory on $S^{1}$ and then on $M$. If we interchange the order of reduction, then we would be reducing a $3 \mathrm{~d} \mathcal{N}=2$ theory on the $S^{1}$ factor of $S^{1} \times_{\varepsilon} D$. This would give an $\Omega$-deformed $\mathcal{N}=(2,2)$ theory on $D[1,26]$. So going back to the original order, we find that the $5 \mathrm{~d}$ MSYM theory

\footnotetext{
${ }^{1}$ This factorization was studied in [15-17] for $k=0,1$ and proved in [18] for $k=1$. The case of general $k$ is discussed in [19]. A similar factorization is expected to hold for the partition functions on $L(k, p)_{b}[11]$.
} 
we obtain is deformed in such a way that it becomes an $\Omega$-deformed $\mathcal{N}=(2,2)$ gauge theory on $D$ upon dimensional reduction on $M$. We call this deformation the $\Omega$-deformation of 5d MSYM theory on $D \times M$.

For the construction of the $\Omega$-deformed 5 d MSYM theory, it is actually more convenient to generalize $S^{1} \times_{\varepsilon} D$ to $S^{1} \times_{V} \Sigma$, where $\Sigma$ is any Riemann surface, and $\times_{V}$ means that the product is twisted with the isometry $\exp (2 \pi R V)$ of $\Sigma$ generated by a Killing vector field $V$. In this more general setup, we must topologically twist the 6 d theory along $\Sigma$ as well in order to preserve some supersymmetry; then $Q$ will be a supercharge of the twisted theory that is a scalar on $\Sigma$ and $M$. As a result, the $\Omega$-deformed 5 d MSYM theory on $\Sigma \times M$ describing the 6 d theory also undergoes topological twisting along $\Sigma$ (on top of the one along $M$ ), and we are interested in the $Q$-invariant sector of this twisted $5 \mathrm{~d}$ theory.

In general, $\mathcal{N}=(2,2)$ gauge theories admit two kinds of topological twist. One is the A-twist which uses the vector R-symmetry $\mathrm{U}(1)_{V}$, and the other is the B-twist which uses the axial R-symmetry $\mathrm{U}(1)_{A}$. We can see which twist is induced on the $5 \mathrm{~d}$ theory by considering the case $\Sigma=\mathbb{R}^{2}$. It has been observed that 5 d MSYM theory on $\mathbb{R}^{2} \times M$ without the $\Omega$-deformation, viewed as an $\mathcal{N}=(2,2)$ gauge theory on $\mathbb{R}^{2}$, has a superpotential given by the Chern-Simons functional for a complex gauge field $\mathcal{A}$ on $M$ [8]. For nonabelian gauge group, the superpotential is not homogeneous in $\mathcal{A}$, and this leads to breaking of $\mathrm{U}(1)_{V}$. So the twisting must be done with $\mathrm{U}(1)_{A}$. To summarize, the dimensional reduction of the $\Omega$-deformed twisted $5 \mathrm{~d}$ MSYM theory on $M$ is an $\Omega$-deformed B-twisted gauge theory. Conversely, we can construct this $5 \mathrm{~d}$ theory by "lifting" an $\Omega$-deformed B-twisted gauge theory from two to five dimensions.

Unlike its A-twisted counterpart [1, 26], the $\Omega$-deformation of B-twisted gauge theories has been little studied in the literature. To achieve our goal, we should therefore understand it first in a general setup, and this is what we try to do in section 2. In [27], the $\Omega$-deformation of B-twisted Landau-Ginzburg models was formulated, and used to provide a unified approach to understanding quantization of the integrable system [28] and the algebra of supersymmetric loop operators [29,30] associated with an $\mathcal{N}=2$ gauge theory in four dimensions. We follow the same strategy as the one employed there, and formulate the $\Omega$-deformation of general B-twisted gauge theories. The construction is relatively straightforward if the worldsheet $\Sigma$ has no boundary. In the situation that $\Sigma$ has a boundary, the supersymmetric action requires an interesting boundary term which turns out to carry much of the information on the dynamics of the theory. We then discuss boundary conditions, and derive a localization formula for correlation functions of $Q$-invariant operators, taking $\Sigma=D$.

In section 3, we turn to the twisted 5d MSYM theory on $\Sigma \times M$. Due to the topological twisting, the theory may be regarded as a B-twisted gauge theory on $\Sigma$. Hence, we can obtain its $\Omega$-deformation by adapting the construction developed in the previous section. For $\Sigma=D$, we show that the twisted theory is equivalent to analytically continued ChernSimons theory on $M$ by localization of the path integral, following essentially the same steps as in the derivation of the $2 \mathrm{~d}$ localization formula.

We conclude our discussion in section 4 by placing the above results in the context of the $3 \mathrm{~d}-3 \mathrm{~d}$ correspondence. We establish the correspondence between $T[M]$ and analytically 
continued Chern-Simons theory described above, and moreover discuss a mirror symmetry between $\Omega$-deformed $\mathcal{N}=(2,2)$ theories in two dimensions.

\section{$2 \Omega$-deformation of B-twisted gauge theories}

In this section we formulate the $\Omega$-deformation of B-twisted gauge theories in two dimensions, and study general properties of the deformed theories. In particular, we derive a localization formula for correlation functions on a disk. The construction developed in this section will be crucial for our discussion in the next section.

\subsection{Supersymmetry transformation laws}

First of all, let us explain what we mean by an $\Omega$-deformation of a B-twisted theory. The notion of $\Omega$-deformation was introduced originally in the context of $\mathcal{N}=2$ gauge theories on $\mathbb{R}^{4}$ [31-35]. The following definition is an analog in the case of B-twisted gauge theories of a more general formulation of $\Omega$-deformation [36], which works for topologically twisted $\mathcal{N}=2$ gauge theories on arbitrary four-manifolds admitting isometries.

After the B-twisting, an $\mathcal{N}=(2,2)$ theory has two supercharges $\bar{Q}_{ \pm}$that are scalars on the worldsheet $\Sigma$. The linear combination $Q=\bar{Q}_{+}+\bar{Q}_{-}$satisfies $Q^{2}=0$ up to a central charge, and is used as the BRST operator of the B-twisted theory. Given a Killing vector field $V$ on $\Sigma$, an $\Omega$-deformation with respect to $V$ is a deformation such that the deformed theory has a BRST operator, which we will still denote by $Q$, satisfying the deformed relation

$$
Q^{2}=L_{V}
$$

Here $L_{V}$ is the conserved charge acting on fields as the gauge-covariant Lie derivative $\mathcal{L}_{V}$ by $V$.

In order to formulate such a deformation, one can start with a supergravity theory and try to find a background that realizes the deformation. For A-twisted theories on $S^{2}$, such a supergravity background was found in [37]. In principle, one can apply a mirror map to this background and obtain the corresponding deformation for B-twisted theories on $S^{2}$. Here we instead follow the strategy employed in [27] for the formulation of $\Omega$-deformed B-twisted Landau-Ginzburg models. So let us first review this strategy.

As we have said above, two of the four supercharges of $\mathcal{N}=(2,2)$ supersymmetry algebra become scalars after the B-twist. The remaining two, on the other hand, become components of a one-form supercharge $G=G_{z} \mathrm{~d} z+G_{\bar{z}} \mathrm{~d} \bar{z}$. Suppose $\Sigma=\mathbb{C}$. Then, these supercharges are all unbroken, and satisfy the commutation relations $\left\{\bar{Q}_{-}, G_{z}\right\}=P_{z}$ and $\left\{\bar{Q}_{+}, G_{\bar{z}}\right\}=P_{\bar{z}}$, where $P=P_{z} \mathrm{~d} z+P_{\bar{z}} \mathrm{~d} \bar{z}$ is the generator for translations. The other commutators vanish, up to central charges.

Now we pick a Killing vector field $V=V^{z} \partial_{z}+V^{\bar{z}} \partial_{\bar{z}}$ and set $Q=\bar{Q}_{+}+\bar{Q}_{-}+\iota_{V} G$, where $\iota_{V}$ is the interior product with $V$. This operator satisfies $Q^{2}=\iota_{V} P$, and this is nothing but the $\Omega$-deformed relation $(2.1)$ on $\mathbb{C}$. Hence, $Q$ generates an $\Omega$-deformed supersymmetry transformation on the flat worldsheet.

What we have to do is to generalize this construction to an arbitrary choice of $\Sigma$ which is not necessarily flat. To this end, we should write down the transformations of fields 
generated by $Q$ in the flat case (see e.g. [38] for the standard formulas for $\mathcal{N}=(2,2)$ supersymmetry transformations), and rewrite them in a way that makes sense even when $\Sigma$ is curved. This is actually not very hard.

A vector multiplet of the B-twisted supersymmetry consists of a gauge field $A$, a oneform $\sigma$, and an auxiliary scalar $\mathbf{D}$, as well as fermionic fields which are two scalars $\bar{\lambda}_{ \pm}$and a one-form $\lambda$. These are all valued in the Lie algebra $\mathfrak{g}$ of the gauge group $G$, except that the gauge field is a connection on a $G$-bundle over $\Sigma$. To avoid introducing dependence on the metric on $\Sigma$ to the supersymmetry transformation laws, in our formulation of the B-twisted gauge theory, we replace $\mathbf{D}$ with a two-form (still called $\mathbf{D}$ ), and $\bar{\lambda}_{ \pm}$by two two-forms $\alpha$ and $\zeta$; these are related to the original fields by the Hodge duality, once a metric is chosen. ${ }^{2}$ Thus, our vector multiplet consists of a gauge field $A$ and

$$
\sigma \in \Omega^{1}(\Sigma ; \mathfrak{g}), \quad \mathbf{D} \in \Omega^{2}(\Sigma ; \mathfrak{g}) ; \quad \lambda \in \Omega^{1}(\Sigma ; \mathfrak{g}), \quad \alpha, \zeta \in \Omega^{2}(\Sigma ; \mathfrak{g}) .
$$

By $\Omega^{p}(\Sigma ; \mathfrak{g})$ we mean the space of $p$-forms in the adjoint representation.

After some rescaling and shifting of fields, we arrive at the following $\Omega$-deformed transformation laws for the vector multiplet:

$$
\begin{aligned}
\delta A & =i \lambda \\
\delta \sigma & =\lambda+\iota_{V} \zeta \\
\delta \lambda & =-i \iota_{V} F_{A}+\mathrm{d}_{A} \iota_{V} \sigma \\
\delta \zeta & =i F_{A}+\mathrm{d}_{A} \sigma-\sigma \wedge \sigma \\
\delta \alpha & =\mathrm{d}_{A} \sigma+\mathbf{D} \\
\delta \mathbf{D} & =\mathrm{d}_{A} \iota_{V} \alpha-\left[\iota_{V} \sigma, \alpha\right]-\mathrm{d}_{A} \lambda-\lambda \wedge \sigma-\sigma \wedge \lambda-\mathrm{d}_{A} \iota_{V} \zeta .
\end{aligned}
$$

Here $\mathrm{d}_{A}=\mathrm{d}-i A$ is the gauge-covariant exterior differential, and $F_{A}$ is the curvature of $A$.

A chiral multiplet consists of fields valued in a unitary representation $R$ of $G$, as well as those valued in the complex conjugate representation $\bar{R}$ which is isomorphic to the dual representation. Those valued in $R$ are a complex scalar $\phi$, a fermionic one-form $\rho$ and an auxiliary two-form $\mathbf{F}$, while those valued in $\bar{R}$ are fermionic scalars $\bar{\eta}$ and $\bar{\theta}$. For the metric independence of supersymmetry transformations, we will use a two-form $\bar{\mu}$ instead of $\bar{\theta}$. Thus, the fields in our chiral multiplet are

$$
\phi \in \Omega^{0}(\Sigma ; R), \quad \mathbf{F} \in \Omega^{2}(\Sigma ; R) ; \quad \bar{\eta} \in \Omega^{0}(\Sigma ; \bar{R}), \quad \rho \in \Omega^{1}(\Sigma ; R), \quad \bar{\mu} \in \Omega^{2}(\Sigma ; \bar{R}) .
$$

The $\Omega$-deformed supersymmetry transformation laws for a chiral multiplet were written down in [27] in the case without coupling to a vector multiplet. It is straightforward to

\footnotetext{
${ }^{2}$ This replacement is necessary for the metric independence even when the $\Omega$-deformation is not present, as can be seen from the transformation laws for $\alpha$ and $\zeta$.
} 
generalize the formula to the gauged case:

$$
\begin{aligned}
\delta \phi & =\iota_{V} \rho, \\
\delta \bar{\phi} & =\bar{\eta}, \\
\delta \rho & =\mathrm{d}_{A} \phi-\sigma \phi+\iota_{V} \mathbf{F}, \\
\delta \bar{\eta} & =\iota_{V} \mathrm{~d}_{A} \bar{\phi}+\bar{\phi} \iota_{V} \sigma, \\
\delta \bar{\mu} & =\overline{\mathbf{F}}, \\
\delta \mathbf{F} & =\mathrm{d}_{A} \rho-\sigma \wedge \rho+\zeta \phi, \\
\delta \overline{\mathbf{F}} & =\mathrm{d}_{A} \iota_{V} \bar{\mu}+\bar{\mu} \iota_{V} \sigma .
\end{aligned}
$$

We let $Q$ denote the generator for the supersymmetry transformations. From the above formulas, one can check that $Q$ squares to $\mathcal{L}_{V}=\iota_{V} \mathrm{~d}_{A}+\mathrm{d}_{A} \iota_{V}$, modulo the gauge transformation generated by $i \iota_{V} \sigma{ }^{3}$ Observables are gauge- and $Q$-invariant operators that are not $Q$-exact. From the supersymmetry transformation laws, we see that gauge-invariant functions of $\phi$, inserted at zeros of $V$, are local observables.

\subsection{Supersymmetric action}

Let us construct an action that is invariant under the $\Omega$-deformed supersymmetry transformations. It takes the form

$$
S=S_{V}+S_{C}+S_{W}
$$

The first two pieces $S_{V}$ and $S_{C}$ contain kinetic terms for the vector and chiral multiplets, respectively, and the last piece $S_{W}$ contains terms constructed from a superpotential $W$, a gauge-invariant holomorphic function of the chiral multiplet scalar $\phi$.

To construct $S_{V}$ and $S_{C}$, we need to pick a complex structure and a Kähler metric on $\Sigma$. We denote the Kähler metric by $h$. Then, the vector multiplet action is

$$
\begin{aligned}
& S_{V}=\delta \int_{\Sigma} \operatorname{Tr}\left(\alpha \wedge \star\left(-\mathrm{d}_{A} \sigma+\mathbf{D}+4 \bar{\partial}_{A} \sigma\right)+\zeta \wedge \star\left(-i F_{A}+\mathrm{d}_{A} \sigma+\sigma \wedge \sigma\right)\right) \\
&=\int_{\Sigma} \operatorname{Tr}( F_{A} \wedge \star F_{A}+\sigma \wedge \star \Delta \sigma+\frac{\kappa}{2} \sigma \wedge \star \sigma-(\sigma \wedge \sigma) \wedge \star(\sigma \wedge \sigma) \\
&+\mathbf{D}^{\prime} \wedge \star \mathbf{D}^{\prime}+2 \partial_{A}\left(\sigma \star \bar{\partial}_{A} \sigma\right)+2 \bar{\partial}_{A}\left(\sigma \star \partial_{A} \sigma\right) \\
&-2 \alpha \wedge \star \mathrm{d}_{A-i \sigma}\left(\lambda^{1,0}-\lambda^{0,1}\right)-2 \zeta \wedge \star \mathrm{d}_{A+i \sigma} \lambda \\
&\left.-\alpha \wedge \star \mathrm{d}_{A-i \sigma} \iota_{V} \alpha-\zeta \wedge \star \mathrm{d}_{A+i \sigma} \iota_{V} \zeta-2 \alpha \wedge \star \mathrm{d}_{A}\left(\left(\iota_{V} \zeta\right)^{1,0}-\left(\iota_{V} \zeta\right)^{0,1}\right)\right),
\end{aligned}
$$

\footnotetext{
${ }^{3}$ More precisely, the supersymmetry transformation laws only show that $Q$ obeys $Q^{2}=L_{V}$ if its action is restricted to fields. Actually, on the right-hand side of this relation, an extra operator may be present that commutes with any fields. Such an operator corresponds to a central charge in the $\mathcal{N}=(2,2)$ supersymmetry algebra. We will not consider this possibility since our discussion only concerns the action of $Q$ on fields.
} 
and the chiral multiplet action is

$$
\begin{aligned}
S_{C}=\delta \int_{\Sigma}\left(\rho \wedge \star\left(\mathrm{d}_{A} \bar{\phi}-\bar{\phi} \sigma+\iota_{V} \overline{\mathbf{F}}\right)-i \phi \bar{\phi} \alpha+\mathbf{F} \wedge \star \bar{\mu}+2 \sigma \phi \wedge \star \iota_{V} \bar{\mu}\right) \\
=\int_{\Sigma}\left(\left(\mathrm{d}_{A} \phi+\iota_{V} \mathbf{F}\right) \wedge \star\left(\mathrm{d}_{A} \bar{\phi}+\iota_{V} \overline{\mathbf{F}}\right)+\sigma \phi \wedge \star(\bar{\phi} \sigma)-i \phi \bar{\phi} \mathbf{D}^{\prime}+\mathbf{F} \wedge \star \overline{\mathbf{F}}\right. \\
\quad-\iota_{V} \mathbf{F} \wedge \star(\bar{\phi} \sigma)+\sigma \phi \wedge \star \iota_{V} \overline{\mathbf{F}}-i \partial(\phi \bar{\phi} \sigma)+i \bar{\partial}(\phi \bar{\phi} \sigma) \\
\quad-\rho \wedge \star \mathrm{d}_{A-i \sigma} \bar{\eta}+\mathrm{d}_{A-i \sigma} \rho \star \bar{\mu}+2 \rho \bar{\phi} \wedge \star \lambda-i \phi \bar{\eta} \alpha+\zeta \phi \star \bar{\mu} \\
\left.\quad+\rho \wedge \star\left(\bar{\phi} \iota_{V} \zeta-\iota_{V} \mathrm{~d}_{A} \iota_{V} \bar{\mu}-\iota_{V} \bar{\mu} \iota_{V} \sigma\right)+2\left(\lambda \phi+\iota_{V} \zeta \phi+\sigma \iota_{V} \rho\right) \wedge \star \iota_{V} \bar{\mu}\right) .
\end{aligned}
$$

Here $\Delta=D^{*} D$ is the Laplacian associated to the covariant derivative $D$ coupled to the gauge field and the Levi-Civita connection, $\kappa$ is the scalar curvature, and $\mathbf{D}^{\prime}=\mathbf{D}+2 \bar{\partial}_{A} \sigma$ is a redefined auxiliary field.

Both $S_{V}$ and $S_{C}$ are $Q$-invariant, provided that $V$ is a Killing vector field. This follows from the fact that $\mathcal{L}_{V}$ commutes with the Hodge star operator $\star$ for such $V$; thanks to this property, we have

$$
\delta^{2} \int_{\Sigma} \mathcal{V}=\int_{\Sigma} \mathcal{L}_{V} \mathcal{V}=\int_{\partial \Sigma} \iota_{V} \mathcal{V}
$$

for any gauge-invariant two-form $\mathcal{V}$ on $\Sigma$ constructed from fields using $\star$, and the last expression vanishes since $\iota_{V} \mathcal{V}$ restricts to zero on $\partial \Sigma$, with $V$ being tangent to $\partial \Sigma$.

The construction of the superpotential term is a little tricky if $\Sigma$ has a boundary. For simplicity, we will assume that $\Sigma$ has only a single connected boundary component. ${ }^{4}$ The boundary is topologically a circle, and we can choose a periodic coordinate $\varphi$ (with period $2 \pi$ ) on the boundary such that

$$
\left.V\right|_{\partial \Sigma}=\varepsilon \partial_{\varphi}
$$

for some real $\varepsilon$. Furthermore, we assume that $V$ generates nontrivial isometries on the boundary, that is, $\varepsilon \neq 0$. Then

$$
S_{W}=i \int_{\Sigma}\left(\mathbf{F} \frac{\partial W}{\partial \phi}+\frac{1}{2} \rho \wedge \rho \frac{\partial^{2} W}{\partial \phi \partial \phi}+\overline{\mathbf{F}} \frac{\partial \bar{W}}{\partial \bar{\phi}}+\bar{\eta} \bar{\mu} \frac{\partial^{2} \bar{W}}{\partial \bar{\phi} \partial \bar{\phi}}\right)-\frac{i}{\varepsilon} \int_{\partial \Sigma} W \mathrm{~d} \varphi
$$

where contraction of gauge indices is implicit. The boundary term is needed for $Q$ invariance.

We impose the reality condition such that $\sigma$ and $\mathbf{D}^{\prime}$ are hermitian, while $\phi^{\dagger}=\bar{\phi}$ and $\mathbf{F}^{\dagger}=\overline{\mathbf{F}}$, so that the real part of the action is nonnegative in the absence of boundary. ${ }^{5}$ If $\Sigma$ has a boundary, we should impose a suitable boundary condition on $\phi$ in order to ensure the convergence of the path integral.

One of the most important features of the action constructed above is that although it depends on the complex structure and the metric of $\Sigma$, the dependence is $Q$-exact. Still, the $\Omega$-deformed B-twisted theory is not quite topological. Rather, it is quasi-topological,

\footnotetext{
${ }^{4}$ If $\Sigma$ has multiple boundary components, then for each component one has a boundary term similar to the one in the formula (2.11).

${ }^{5}$ This is true even when $\kappa<0$ since the bosonic part of $S_{V}$ can be written as the integral of $\operatorname{Tr}\left(\left(F_{A}+\right.\right.$ $\left.i \sigma \wedge \sigma) \wedge \star\left(F_{A}+i \sigma \wedge \sigma\right)+4 \partial_{A} \sigma \wedge \star \bar{\partial}_{A} \sigma+\mathbf{D}^{\prime} \wedge \star \mathbf{D}^{\prime}\right)$, which is manifestly nonnegative.
} 
in the sense that it is invariant under deformations of the complex structure and the metric as long as $V$ remains as a Killing vector field.

So far $V$ has been assumed to be a real vector field. We can relax this condition and multiply $V$ by a phase factor, since the action remains $Q$-invariant and nonnegative if we simply replace the appearance of $V$ by its complex conjugate $\bar{V}$ in the first line of the formula (2.8) for $S_{C}$. A phase rotation of $V$ is actually equivalent to the opposite phase rotation of $W$, for the former has the same effect as the latter combined with the action of an element in the vector R-symmetry group $\mathrm{U}(1)_{V}$ (with the chiral multiplet assigned charge 0 under it), but the $\mathrm{U}(1)_{V}$-action can be undone by a field redefinition (which does not modify the path integral measure, as there is no quantum anomaly for $\left.\mathrm{U}(1)_{V}\right)$.

\subsection{Boundary condition}

We have constructed the $\Omega$-deformed B-twisted theory on a general worldsheet $\Sigma$. In particular, we allowed the possibility that $\Sigma$ has a boundary. We now discuss boundary conditions.

The boundary of $\Sigma$ is topologically a circle, and the Killing vector field $V$ generates its rotations. The neighborhood of the boundary looks like a short cylinder. We equip this cylinder with a flat metric $\mathrm{d} s^{2}=\mathrm{d} n^{2}+\mathrm{d} \varphi^{2}$, with $n$ being a coordinate in the direction normal to the boundary. After the boundary condition is fixed, one can deform the metric of $\Sigma$ to anything that is allowed by the quasi-topological property of the theory. However, the boundary condition will depend on the initial choice of the flat metric in the neighborhood of the boundary.

Our boundary conditions must meet two requirements. One is that they should lead to a good variational problem in a semiclassical, or weak coupling, limit. In our case there is a natural weak coupling limit, which is obtained by rescaling the $Q$-exact part of the action by a large factor; correlation functions of $Q$-invariant operators are left unchanged under such a $Q$-exact deformation. So we require that boundary terms be absent in the variation of the action when we vary the fields in this limit. The other requirement is that boundary conditions must be $Q$-invariant so that $Q$ preserves the space of allowed field configurations.

We first analyze boundary conditions for the vector multiplet fields. The gauge field has the standard kinetic term, so its boundary condition is a standard one, namely either the Dirichlet or Neumann boundary condition. Since a gauge-invariant expression for the former condition does not exist in two dimensions, we choose the latter, $F_{n \varphi}=0$. Gauging $A_{n}$ away, we can write this condition as $\partial_{n} A_{\varphi}=0$. The requirement of $Q$-invariance then leads to $\partial_{n} \sigma_{\varphi}=\lambda_{n}=\partial_{n} \lambda_{\varphi}=0$. If we now look at the kinetic term for $\sigma$ in the vector multiplet action (2.7), we notice that it differs from the standard one by total derivative terms. A natural way to kill these unwanted terms is to set $\sigma_{n}=0$ on the boundary; the total derivative terms in the chiral multiplet action (2.8) also drop out then. Taking the $Q$-variation of this condition, we get $\zeta_{n \varphi}=0$.

In fact, the set of boundary conditions we have found so far is part of the conditions imposed by a B-brane in $\mathcal{N}=(2,2)$ gauge theory [39,40]. This suggests that we should 
choose our boundary condition for the vector multiplet to be the B-brane condition:

$$
A_{n}=\partial_{n} A_{\varphi}=\sigma_{n}=\partial_{n} \sigma_{\varphi}=\lambda_{n}=\partial_{n} \lambda_{\varphi}=\zeta_{n \varphi}=\partial_{n} \alpha_{n \varphi}=\partial_{n} \mathbf{D}_{n \varphi}^{\prime}=0 .
$$

This set of boundary conditions is not $Q$-invariant by itself. In order to achieve $Q$ invariance, we further impose an infinite series of conditions, generated from the above conditions by the action of even powers of $\partial_{n}$ [39].

We stress that the gauge $A_{n}=0$ has been chosen on the boundary above. For compatibility, we must restrict gauge transformations to be such that their parameters have vanishing normal derivatives on the boundary. In addition, we can impose a restriction on the boundary values of gauge transformations. To do so, we pick a subgroup $H$ of $G$ and require gauge transformations to be valued in $H$ on the boundary. Then, those gauge transformations that do not satisfy this condition form a physical symmetry of the theory, provided that they leave invariant the boundary condition for the chiral multiplet, to which we now turn.

Thanks to the condition $A_{n}=0$, the boundary terms arising from variation of the chiral multiplet action (2.8) are all independent of the vector multiplet fields. Furthermore, the supersymmetry variations for $\phi$ and $\bar{\phi}$ do not depend on vector multiplet fields either. In this situation, the analysis of the boundary condition for the chiral multiplet reduces to the case of Landau-Ginzburg models [27]. Hence, we can impose the same boundary condition as in that case.

We refer the reader to [27] for the details of the analysis, and here simply state the result. The boundary condition for the chiral multiplet depends on a choice of a submanifold $\gamma$ in the target space, which may be considered as the support of a brane of a certain type. Then the scalars obey the usual D-brane boundary condition:

$$
\phi \in \gamma, \quad \partial_{n} \phi \in N_{\mathbb{R}} \gamma
$$

at each point on $\partial \Sigma$, where $N_{\mathbb{R}} \gamma$ is the normal bundle of $\gamma$. The fermions obey

$$
\left(\varepsilon \rho_{\varphi}, \bar{\eta}\right) \in T_{\mathbb{C}} \gamma, \quad\left(\varepsilon \rho_{n}, \bar{\mu}_{n \varphi}\right) \in N_{\mathbb{C}} \gamma
$$

Again, there are further conditions obtained by repeated action of $Q$ on the above conditions, which guarantee that the boundary condition is $Q$-invariant.

The target space for the chiral multiplet is the representation space $V_{R}$ of $R$, equipped with the $G$-invariant Kähler form

$$
\omega=i \mathrm{~d} \phi \wedge \mathrm{d} \bar{\phi}
$$

Note that here $\phi=\left(\phi^{1}, \ldots, \phi^{\operatorname{dim} V_{R}}\right)$ is considered as a set of complex coordinates on $V_{R}$; thus $\mathrm{d} \phi$ is a set of $(1,0)$-forms on $V_{R}$. We require $\gamma$ to be a Lagrangian submanifold of the symplectic manifold $\left(V_{R}, \omega\right)$. As we will see, this has the effect of eliminating fermion zero modes.

Moreover, $\gamma$ must be $H$-invariant for gauge invariance to be unbroken. This requirement has the following consequence. Let $\left\{T_{a}\right\}$ be a set of generators of $G$, and $X_{a}$ denote 
the vector fields on $V_{R}$ generated by the action of $T_{a}$. The moment map $\mu: V_{R} \rightarrow \mathfrak{g}^{\vee}$ for the $G$-action on the symplectic manifold $\left(V_{R}, \omega\right)$ is given by

$$
\left(\mu, T_{a}\right)=i \phi \bar{\phi} T_{a} .
$$

Let $\mu_{H}: V_{R} \rightarrow \mathfrak{h}^{\vee}$ be the moment map for the $H$-action; by definition, $\left(\mathrm{d} \mu_{H}, T_{a}\right)=\iota_{X_{a}} \omega$ for $T_{a} \in \mathfrak{h}$. Since $\gamma$ is $H$-invariant and a Lagrangian submanifold by assumption, $X_{a}$ are tangent to $\gamma$ and $\iota_{X} \omega$ vanishes on $T \gamma$. It follows that $\mu_{H}$ is constant on the boundary, as any variation of $\phi$ is tangent to $\gamma$ due to the boundary condition $\phi \in \gamma$.

\subsection{Localization}

Finally, we derive a formula for correlation functions of $Q$-invariant operators via localization of the path integral. We take our worldsheet $\Sigma$ to be a disk $D$, and equip it with a rotationally invariant metric $h$. The Killing vector field $V$ generates rotations and can be written as $V=\varepsilon \partial_{\varphi}$ for some $\varepsilon \neq 0$. By the quasi-topological property of the theory, we can always deform $h$ into a metric with scalar curvature $\kappa>0$, such as one for a hemisphere. We choose the subgroup $H$ to be trivial, that is, we choose to divide the field space by gauge transformations that equal the identity on the boundary.

In order to localize the path integral, one usually rescales the $Q$-exact part of the action by a large factor $t$, which in our case means rescaling $S_{V}+S_{C} \rightarrow t\left(S_{V}+S_{C}\right)$. On the other hand, we expect that the theory simplifies considerably when $D$ is very small, since in such a situation most degrees of freedom are very massive and decouple from the dynamics. So we may also want to rescale the metric as $h \rightarrow t^{-2} h$. If we combine these two ways to simplify the path integral, the net effect is that $S_{V}$ is rescaled by a factor of $t^{3}$, while $S_{C}$ is rescaled by a factor of $t$, except the term coming from $F \wedge \star \bar{\mu}$ in the $Q$-exact expression (2.8) which is rescaled by $t^{3}$. Motivated by this consideration, we deform the action as follows:

$$
S \rightarrow t^{3}\left(S_{V}-\delta \int_{D} \operatorname{Tr} \alpha \star \mathbf{D}^{\prime}\right)+t\left(S_{C}+s \delta \int_{D} \mathbf{F} \star \bar{\mu}\right)+S_{W} .
$$

Here $s$ is a real parameter.

First, we rescale $\bar{\mu} \rightarrow s^{-1} \bar{\mu}$ and take the limit $s \rightarrow \infty$. In this limit, integrating out the auxiliary field $\mathbf{F}$ is equivalent to simply setting

$$
\mathbf{F}=0 .
$$

The term containing $\mathbf{D}^{\prime}$ is included in the deformation so that integrating $\mathbf{D}^{\prime}$ out produces delta functions imposing the constraint

$$
\mu=-t^{2} \star\left(\partial_{A} \sigma-\bar{\partial}_{A} \sigma\right),
$$

where the moment map $\mu$ is given by the formula (2.16), and $\sigma$ is regarded as valued in $\mathfrak{g}^{\vee}$ by $(\sigma, X)=\operatorname{Tr}(\sigma X)$ for $X \in \mathfrak{g}$.

Next, we take $t$ to be large (but still finite). Looking at the bosonic parts of $S_{V}$ and $S_{C}$, we find that the path integral then localizes, under the boundary condition (2.12), to the locus given by

$$
F_{A}=\sigma=\mathrm{d}_{A} \phi=0
$$


As $D$ is simply connected, the equation $F_{A}=0$ means that we can set

$$
A=0
$$

everywhere by a gauge transformation. Together with the boundary condition (2.13) and the constraint (2.19), the equations $\sigma=\mathrm{d}_{A} \phi=0$ then imply that the path integral localizes to the configurations where $\phi$ is a constant map to the subspace $\gamma \cap \mu^{-1}(0)$ of the target space $V_{R}$.

Since the path integral localizes for large $t$, we can evaluate it by perturbation theory (in $1 / \sqrt{t}$ ) around background configurations on the localization locus. We will denote backgrounds with subscript 0 and fluctuations around them with a tilde; thus $A=\widetilde{A}$, $\sigma=\tilde{\sigma}$, and $\phi=\phi_{0}+\tilde{\phi}$.

For the computation we need to fix the gauge. We choose the standard gauge-fixing condition $\nabla_{\mu} A^{\mu}=0$ and add to the action the gauge-fixing term

$$
S_{G}=t^{3} \int_{D} \sqrt{h} \mathrm{~d}^{2} x \operatorname{Tr}\left(\bar{c} \nabla^{\mu} D_{\mu} c+\left(\nabla^{\mu} A_{\mu}\right)^{2}\right),
$$

where $\nabla$ is the Levi-Civita connection and $c, \bar{c}$ are ghosts. After rescaling the fluctuations and the fermions as

$$
\begin{aligned}
(\widetilde{A}, \tilde{\sigma}, \lambda, \zeta, \alpha) & \rightarrow\left(t^{-3 / 2} \widetilde{A}, t^{-3 / 2} \tilde{\sigma}, t^{-1} \lambda, t^{-2} \zeta, t^{-2} \alpha\right), \\
(\tilde{\phi}, \rho, \bar{\eta}, \bar{\mu}) & \rightarrow\left(t^{-1 / 2} \tilde{\phi}, t^{-1} \rho, \bar{\eta}, \bar{\mu}\right), \\
(c, \bar{c}) & \rightarrow\left(t^{-3 / 2} c, t^{-3 / 2} \bar{c}\right),
\end{aligned}
$$

the terms in the action containing them become

$$
\begin{aligned}
\int_{D}\left(\operatorname { T r } \left(\widetilde{A} \wedge \star \Delta_{\mathrm{d}} \widetilde{A}+\tilde{\sigma} \wedge \star \Delta_{\mathrm{d}} \tilde{\sigma}+\alpha \wedge \star(\right.\right. & \left.\partial \lambda-\bar{\partial} \lambda)-2 \zeta \wedge \star \mathrm{d} \lambda+\bar{c} \wedge \star \Delta_{\mathrm{d}} c\right) \\
& +\mathrm{d} \tilde{\phi} \wedge \star \mathrm{d} \tilde{\bar{\phi}}-\rho \wedge \star \mathrm{d} \bar{\eta}+\mathrm{d} \rho \wedge \star \bar{\mu}+\cdots) .
\end{aligned}
$$

Here $\Delta_{\mathrm{d}}=\left(\mathrm{d}+\mathrm{d}^{*}\right)^{2}$ is the Hodge-de Rham laplacian, and the ellipsis refers to terms multiplied by negative powers of $t$. To obtain this expression we have used the relation $\Delta_{\mathrm{d}}=\nabla^{*} \nabla+\kappa / 2$ in the space of one-forms on a surface.

We have to integrate over the fluctuations and the fermions. To do this, we deform $D$ into the shape of a two-sphere $S^{2}$ with a small disk $D_{\epsilon}$ of radius $\epsilon$ removed. Since fields on $S^{2} \backslash D_{\epsilon}$ can be obtained from fields on $S^{2}$ by restriction, we can expand them in the eigenmodes of $\Delta_{\mathrm{d}}$ on $S^{2}{ }^{6}$ The integral (2.24), when expressed in terms of the expansion coefficients, differs from the case with $\Sigma=S^{2}$ by $\epsilon$-dependent terms. However, at the end of the localization computation, we can take the limit $\epsilon \rightarrow 0$ (which is a $Q$-exact operation), whereby the difference simply vanishes. Thus, we can perform the integration

\footnotetext{
${ }^{6} \mathrm{On} S^{2}$, the fermionic part of the leading terms in the integral (2.24) can be written as

$$
-\left\langle\rho,\left(\mathrm{d}+\mathrm{d}^{*}\right)(\bar{\eta}+\bar{\mu})\right\rangle+\left\langle\alpha-2 \zeta,\left(\mathrm{d}+\mathrm{d}^{*}\right) \lambda^{0,1}\right\rangle-\left\langle\alpha+2 \zeta,\left(\mathrm{d}+\mathrm{d}^{*}\right) \lambda^{1,0}\right\rangle+\left\langle\bar{c}, \Delta_{\mathrm{d}} c\right\rangle,
$$
}

using an appropriate inner product $\langle$,$\rangle . It is thus natural to expand the fermions in the eigenmodes of \Delta_{\mathrm{d}}$. 
over the fluctuations and the fermions in a way similar to the $S^{2}$ case. The computation is not quite like that case, however, since the boundary condition imposes relations among the expansion coefficients.

To understand the result of the integration, we note the following three points. First, the leading terms in the integral (2.24) are completely independent of the background. Second, the boundary condition does not depend on the background either. This is because the support $\gamma$ of the brane is a Lagrangian submanifold of $V_{R}$, and the tangent bundles at different points on $\gamma$ are all isomorphic up to unitary rotations which are symmetries of the action. Finally, there are no fermion zero modes, as we will see shortly; they are all eliminated by the boundary condition. Hence, to leading order, the integration over the fluctuations and the fermions just produces a constant independent of the background, though it may depend on the choice of $\gamma$ and the representation $R$.

Once the perturbative computation is carried out, we integrate over the localization locus. On this locus, the only surviving piece of the action is the boundary term in the superpotential term (2.11):

$$
-\frac{i}{\varepsilon} \int_{\partial D} W \mathrm{~d} \varphi=-\frac{2 \pi i}{\varepsilon} W .
$$

Finally, taking the limit $t \rightarrow \infty$ whereby the subleading terms vanish, we conclude that the correlation function on the disk of any $Q$-invariant operator $\mathcal{O}$ is given by the formula

$$
\langle\mathcal{O}\rangle=\int_{\gamma \cap \mu^{-1}(0)} \mathrm{d} \phi_{0} \exp \left(\frac{2 \pi i}{\varepsilon} W\right) \mathcal{O} .
$$

From this formula we see that the nontrivial information on the dynamics on $D$ is encoded in the boundary term.

In the above derivation, we have asserted that there are no fermion zero modes. Let us show this now. Recall that we have expanded the fermions in the eigenmodes of the Laplacian on $S^{2}$. There are no harmonic one-forms on $S^{2}$, so there are no zero modes for $\lambda$ and $\rho$. Furthermore, harmonic two-forms are Hodge duals of constants, and neither constant $\star \alpha$ nor $\star \zeta$ is compatible with the boundary condition $\partial_{n} \alpha_{n \varphi}=\zeta_{n \varphi}=0$. (To see this for $\alpha$, suppose that we equip $D$ with the metric $\left(\mathrm{d} n^{2}+n^{2} \mathrm{~d} \varphi^{2}\right) /\left(1+n^{2}\right)^{2}$ of the Riemann sphere parametrized by $z=n e^{i \varphi}$, where $(n, \varphi)$ are the cylindrical coordinates used in describing the boundary condition, with the boundary located at $n=0$. Then, the zero mode of $\alpha$ behaves as $\alpha_{n \varphi} \sim n /\left(1+n^{2}\right)^{2}$ near the boundary.) The boundary condition (2.14), on the other hand, implies that the zero mode parts $\bar{\eta}_{0}, \bar{\mu}_{0}$ of $\bar{\eta}, \bar{\mu}$, obey $\left(0, \bar{\eta}_{0}\right) \in T_{\mathbb{C}} \gamma$ and $\left(0, \star \bar{\mu}_{0}\right) \in N_{\mathbb{C}} \gamma$ on the boundary. ${ }^{7}$ Since $\gamma$ is a Lagrangian submanifold of a Kähler manifold for which the complex structure exchanges the tangent and normal bundles, it follows that $\bar{\eta}_{0}=\bar{\mu}_{0}=0$ on the boundary and hence everywhere. So there are no zero modes for $\bar{\eta}$ and $\bar{\mu}$, either. Lastly, the zero modes for the ghosts $c, \bar{c}$ are constant, but there are no such modes to begin with. This is a consequence of our choice to divide

\footnotetext{
${ }^{7}$ To be precise, the boundary condition is imposed on the fermionic fields themselves and not just on their zero modes. However, if we take the limit such that the radius of the $S^{2}$ goes to zero, all nonzero modes become infinitely massive and decouple. Then the fermions may be replaced by their zero mode parts, and the boundary condition is written entirely in terms of the zero modes.
} 
the field space by gauge transformations that equal the identity on the boundary; gauge transformation parameters must vanish on the boundary, therefore so do the ghosts.

\section{Chern-Simons theory from 5d MSYM theory}

As an application of the formulation developed above, in this section we construct an $\Omega$ deformation of $5 \mathrm{~d}$ MSYM theory, placed and topologically twisted on $\Sigma \times M$, where $M$ is a three-manifold. This is achieved by "lifting" the supersymmetry transformation laws and the supersymmetric action constructed in the previous section from two to five dimensions. Then, we show that when $\Sigma$ is a disk $D$, the $\Omega$-deformed twisted 5 d MSYM theory is equivalent to analytically continued Chern-Simons theory on $M$, with integration contour specified by the boundary condition of the $5 \mathrm{~d}$ theory. These results will be the bases for our derivations of various correspondences presented in the next section.

\section{$3.1 \Omega$-deformed twisted 5d MSYM theory on $\Sigma \times M$}

To begin, we formulate the $\Omega$-deformation of the twisted 5 d MSYM theory on $\Sigma \times M$. The gauge group is a compact Lie group $G$. We equip the Riemann surface $\Sigma$ with a metric $h_{\Sigma}$ and $M$ with a metric $h_{M}$, and choose a Killing vector field $V$ generating isometries of $\Sigma$. The metric on $\Sigma \times M$ is $h=h_{\Sigma} \oplus h_{M}$. We write $\left(x^{M}\right)=\left(x^{\mu}, x^{m}\right)$ for coordinates on $\Sigma \times M$.

The theory is topologically twisted as follows. The structure group of the spinor bundle of $\Sigma \times M$ is $\operatorname{Spin}(2)_{\Sigma} \times \operatorname{Spin}(3)_{M} \cong \mathrm{U}(1)_{\Sigma} \times \mathrm{SU}(2)_{M}$. Correspondingly, we split the R-symmetry group $\operatorname{Spin}(5)_{R}$ as $\operatorname{Spin}(2)_{R} \times \operatorname{Spin}(3)_{R} \cong \mathrm{U}(1)_{R} \times \mathrm{SU}(2)_{R}$. The field content of the untwisted theory consists of a gauge field $A$, five scalars $X$, and fermions $\Psi$, transforming under $\mathrm{SU}(2)_{M} \times \mathrm{SU}(2)_{R} \times \mathrm{U}(1)_{\Sigma} \times \mathrm{U}(1)_{R}$ as

$$
\begin{aligned}
& A:(\mathbf{1}, \mathbf{1})^{( \pm 2,0)} \oplus(\mathbf{3}, \mathbf{1})^{(0,0)}, \\
& X:(\mathbf{1}, \mathbf{1})^{(0, \pm 2)} \oplus(\mathbf{1}, \mathbf{3})^{(0,0)}, \\
& \Psi:(\mathbf{2}, \mathbf{2})^{( \pm 1, \pm 1)} .
\end{aligned}
$$

First, we replace $\mathrm{SU}(2)_{M}$ with the diagonal subgroup $\mathrm{SU}(2)_{M}^{\prime}$ of $\mathrm{SU}(2)_{M} \times \mathrm{SU}(2)_{R}$. Under $\mathrm{SU}(2)_{M}^{\prime} \times \mathrm{U}(1)_{\Sigma} \times \mathrm{U}(1)_{R}$, the fields transform as

$$
\begin{aligned}
& A: \mathbf{1}^{( \pm 2,0)} \oplus \mathbf{3}^{(0,0)}, \\
& X: \mathbf{1}^{(0, \pm 2)} \oplus \mathbf{3}^{(0,0)}, \\
& \Psi: \mathbf{1}^{( \pm 1, \pm 1)} \oplus \mathbf{3}^{( \pm 1, \pm 1)} .
\end{aligned}
$$

From the transformation property of $\Psi$, we see that the theory now has $\mathcal{N}=(2,2)$ supersymmetry on $\Sigma$. Next, we identify $\mathrm{U}(1)_{R}$ with the axial R-symmetry group $\mathrm{U}(1)_{A}$, and perform the B-twist on $\Sigma$, replacing $\mathrm{U}(1)_{\Sigma}$ with the diagonal subgroup of $\mathrm{U}(1)_{\Sigma} \times \mathrm{U}(1)_{R}$.

In the language of $\mathcal{N}=(2,2)$ supersymmetry on $\Sigma$, the fields of the twisted $5 \mathrm{~d}$ MSYM theory are grouped into a vector multiplet that is a scalar on $M$, and three adjoint-valued chiral multiplets that combine into a one-form on $M$. (Recall, however, that some of the 
fermions are redefined in our construction.) The scalars of the chiral multiplets are complex combinations of the components $A_{m}$ of $A$ along $M$ and three scalars $X_{m}$ :

$$
\mathcal{A}_{m}=A_{m}+i X_{m}, \quad \overline{\mathcal{A}}_{m}=A_{m}-i X_{m}
$$

These can be regarded as components of a complex gauge field $\mathcal{A}=\mathcal{A}_{m} \mathrm{~d} x^{m}$ on $M$ and its hermitian conjugate $\overline{\mathcal{A}}$.

Being a B-twisted gauge theory, the $\Omega$-deformation of the twisted $5 \mathrm{~d}$ MSYM theory can be formulated in a way similar to the construction discussed in the previous section. To adapt, or "lift," that construction to the present $5 \mathrm{~d}$ setup, we just need to replace every appearance of $-i A_{m}$ in our formulas with the covariant derivative $D_{m}=\nabla_{m}-i A_{m}$ with respect to $A_{m}$ and the Levi-Civita connection on $M$; the replacement makes the formulas invariant under $5 \mathrm{~d}$ gauge transformations, and provides derivatives along $M$. Actually, only the combinations $-i \mathcal{A}_{m}$ and $-i \overline{\mathcal{A}}_{m}$ appear, and these are replaced with $\mathcal{D}_{m}=D_{m}+X_{m}$ and $\overline{\mathcal{D}}_{m}=D_{m}-X_{m}$, respectively.

For those fields that are in the vector multiplet on $\Sigma$, the lifted supersymmetry transformation laws take the same form (2.3) as before, the only difference being that the fields can now depend on the position on $M$. In components, the formula reads

$$
\begin{aligned}
\delta A_{\mu}= & i \lambda_{\mu} \\
\delta \sigma_{\mu}= & \lambda_{\mu}+V^{\nu} \zeta_{\nu \mu}, \\
\delta \lambda_{\mu}= & -i V^{\nu} F_{\nu \mu}+D_{\mu}\left(V^{\nu} \sigma_{\nu}\right) \\
\delta \zeta_{\mu \nu}= & i F_{\mu \nu}+D_{\mu} \sigma_{\nu}-D_{\nu} \sigma_{\mu}-\left[\sigma_{\mu}, \sigma_{\nu}\right] \\
\delta \alpha_{\mu \nu}= & D_{\mu} \sigma_{\nu}-D_{\nu} \sigma_{\mu}+\mathbf{D}_{\mu \nu} \\
\delta \mathbf{D}_{\mu \nu}= & D_{\mu}\left(V^{\rho} \alpha_{\rho \nu}\right)-D_{\nu}\left(V^{\rho} \alpha_{\rho \mu}\right)-\left[V^{\rho} \sigma_{\rho}, \alpha_{\mu \nu}\right] \\
& -D_{\mu} \lambda_{\nu}+D_{\nu} \lambda_{\mu}-\left[\lambda_{\mu}, \sigma_{\nu}\right]-\left[\sigma_{\mu}, \lambda_{\nu}\right]-D_{\mu}\left(V^{\rho} \zeta_{\rho \nu}\right)+D_{\nu}\left(V^{\rho} \zeta_{\rho \mu}\right) .
\end{aligned}
$$

The supersymmetry transformation laws for the chiral multiplets are lifted to

$$
\begin{aligned}
\delta \mathcal{A}_{m} & =V^{\mu} \rho_{\mu m}, \\
\delta \overline{\mathcal{A}}_{m} & =\bar{\eta}_{m}, \\
\delta \rho_{\mu m} & =F_{\mu m}+i D_{\mu} X_{m}+i \mathcal{D}_{m} \sigma_{\mu}+V^{\nu} \mathbf{F}_{\nu \mu m}, \\
\delta \bar{\eta}_{m} & =V^{\mu}\left(F_{\mu m}-i D_{\mu} X_{m}\right)+i V^{\mu} \overline{\mathcal{D}}_{m} \sigma_{\mu}, \\
\delta \bar{\mu}_{\mu \nu m} & =\overline{\mathbf{F}}_{\mu \nu m}, \\
\delta \mathbf{F}_{\mu \nu m} & =D_{\mu} \rho_{\nu m}-D_{\nu} \rho_{\mu m}-\left[\sigma_{\mu}, \rho_{\nu m}\right]+\left[\sigma_{\nu}, \rho_{\mu m}\right]-i \mathcal{D}_{m} \zeta_{\mu \nu}, \\
\delta \overline{\mathbf{F}}_{\mu \nu m} & =D_{\mu}\left(V^{\rho} \bar{\mu}_{\rho \nu m}\right)-D_{\nu}\left(V^{\rho} \bar{\mu}_{\rho \mu m}\right)+\bar{\mu}_{\mu \nu m} V^{\rho} \sigma_{\rho} .
\end{aligned}
$$

Typical observables are gauge-invariant operators constructed from $\mathcal{A}$, such as Wilson lines, inserted at zeros of $V$ on $\Sigma$.

Likewise, we can lift the supersymmetric action from two dimensions. The result is

$$
\begin{aligned}
S_{V}=\int_{\Sigma \times M} \sqrt{h} \mathrm{~d}^{5} x \operatorname{Tr}\left(\frac{1}{2} F_{\mu \nu} F^{\mu \nu}+D_{\mu} \sigma_{\nu} D^{\mu} \sigma^{\nu}+\frac{\kappa}{2} \sigma^{\mu} \sigma_{\mu}\right. \\
\left.-\frac{1}{2}\left[\sigma_{\mu}, \sigma_{\nu}\right]\left[\sigma^{\mu}, \sigma^{\nu}\right]+\frac{1}{2} \mathbf{D}_{\mu \nu}^{\prime} \mathbf{D}^{\prime \mu \nu}+\cdots\right)
\end{aligned}
$$


for the vector multiplet, and

$$
\begin{aligned}
& S_{C}=\int_{\Sigma \times M} \sqrt{h} \mathrm{~d}^{5} x \operatorname{Tr}(\left(F_{\mu m}+i D_{\mu} X_{m}+V^{\nu} \mathbf{F}_{\nu \mu m}\right)\left(F^{\mu m}-i D^{\mu} X^{m}+V^{\nu} \overline{\mathbf{F}}_{\nu}^{\mu m}\right) \\
&+\mathcal{D}_{m} \sigma_{\mu} \overline{\mathcal{D}}^{m} \sigma^{\mu}-i D^{m} X_{m} \mathbf{D}^{\prime \mu \nu} \epsilon_{\mu \nu}+\frac{1}{2} \mathbf{F}_{\mu \nu m} \overline{\mathbf{F}}^{\mu \nu m} \\
&\left.\quad-i V^{\nu} \mathbf{F}_{\nu \mu m} \overline{\mathcal{D}}^{m} \sigma^{\mu}-i V^{\nu} \overline{\mathbf{F}}_{\nu \mu m} \mathcal{D}^{m} \sigma^{\mu}+\cdots\right)
\end{aligned}
$$

for the chiral multiplet. Here $\epsilon_{\mu \nu}$ are components of the volume form $\sqrt{h_{\Sigma}} \mathrm{d}^{2} x$ on $\Sigma$, and we have abbreviated boundary terms and fermionic terms. For the superpotential term, the form of $S_{W}$ is the same as in the $2 \mathrm{~d}$ case, with $W$ now being a gauge-invariant holomorphic functional of the complex gauge field $\mathcal{A}$ on $M$.

The action for the $\Omega$-deformed twisted $5 \mathrm{~d}$ MSYM theory is the sum

$$
S=\frac{1}{2 e^{2}}\left(S_{V}+S_{C}+S_{W}\right)
$$

where $e^{2}$ is the coupling constant of the theory. The question is what superpotential is the right one to use.

Note that neither $S_{V}$ nor $S_{C}$ described above contains kinetic terms for $A_{m}$ and $X_{m}$ along $M$. So these terms should be generated by the superpotential. Since the potential associated with $\mathbf{F}$ is proportional to $|\partial W / \partial \mathcal{A}|^{2}$ and the kinetic terms for $A_{m}$ and $X_{m}$ are of second order in derivatives, $W$ must be of first order. A natural candidate is then the Chern-Simons functional for $\mathcal{A}$. It turns out that the right choice is [8]

$$
W=\frac{1}{2} \int_{M} \operatorname{Tr}\left(\mathcal{A} \wedge \mathrm{d} \mathcal{A}-\frac{2 i}{3} \mathcal{A} \wedge \mathcal{A} \wedge \mathcal{A}\right) .
$$

For this choice of $W$, the superpotential term (2.11) is given by

$$
\begin{array}{r}
S_{W}=\frac{i}{2} \int_{\Sigma \times M} \sqrt{h} \mathrm{~d}^{5} x \operatorname{Tr}\left(\frac{1}{2} \mathbf{F}_{\mu \nu l} \mathcal{F}_{m n}+\rho_{\mu l} \mathcal{D}_{m} \rho_{\nu n}+\frac{1}{2} \overline{\mathbf{F}}_{\mu \nu l} \overline{\mathcal{F}}_{m n}+\bar{\eta}_{l} \overline{\mathcal{D}}_{m} \bar{\mu}_{\mu \nu n}\right) \epsilon^{\mu \nu l m n} \\
-\frac{i}{\varepsilon} \int_{\partial \Sigma} W \mathrm{~d} \varphi
\end{array}
$$

To see that the above choice of $W$ is indeed the right one, set $V=0$ and integrate out the auxiliary fields. Integrating out $\mathbf{D}^{\prime}$ gives the potential $\operatorname{Tr}\left(D_{m} X^{m}\right)^{2}$, while integrating out $\mathbf{F}$ produces $\frac{1}{2} \operatorname{Tr} \mathcal{F}_{m n} \overline{\mathcal{F}}^{m n}$, where $\mathcal{F}_{m n}$ are components of the curvature of $\mathcal{A}$. Up to a total derivative on $M$ (which, for $M=\mathbb{R}^{3}$, vanishes upon integration under usual boundary conditions), the two contributions combine to give

$$
\operatorname{Tr}\left(\frac{1}{2} F_{m n} F^{m n}+D_{m} X_{n} D^{m} X^{n}-\frac{1}{2}\left[X_{m}, X_{n}\right]\left[X^{m}, X^{n}\right]\right) .
$$

After this is done, the bosonic part of the action can be written as

$$
\frac{1}{2 e^{2}} \int_{\mathbb{R}^{5}} \mathrm{~d}^{5} x \operatorname{Tr}\left(\frac{1}{2} F_{M N} F^{M N}+D_{M} X_{N} D^{M} X^{N}-\frac{1}{2}\left[X_{M}, X_{N}\right]\left[X^{M}, X^{N}\right]\right)
$$


for flat spacetime $\mathbb{R}^{5}$, with $\left(X_{M}\right)=\left(\sigma_{\mu}, X_{m}\right)$. This is precisely the bosonic part of the standard 5d MSYM action. ${ }^{8}$

Although the Chern-Simons superpotential (3.9) correctly reproduces the 5d MSYM action, it also causes a problem in the case that $\Sigma$ has a boundary. The real part of the Chern-Simons functional for $\mathcal{A}$ shifts by integer multiples of $2 \pi$ under gauge transformations that are not connected to the identity. Since the Chern-Simons functional enters the boundary term in $S_{W}$, this would mean that the action is gauge invariant modulo $2 \pi i$ if and only if $1 / \varepsilon$ obeys a certain quantization condition, and otherwise the path integral would not be well-defined. However, we do not want to restrict the possible values of $\varepsilon$. So we instead restrict the gauge symmetry — on the boundary, we only allow topologically trivial gauge transformations.

The lifted formulas for $S_{V}$ and $S_{C}$ are $Q$-exact. Hence, the quasi-topological property of the $\Omega$-deformed theory discussed in the $2 \mathrm{~d}$ context still holds for the theory constructed here. In addition, the theory is topological on $M$, since the metric on $M$ enters the action only through $S_{V}$ and $S_{C}$.

The reader might worry that our twisted 5d MSYM theory may not be well-defined. Indeed, $5 \mathrm{~d}$ gauge theories are in general not perturbatively renormalizable by the standard argument. Despite its highly supersymmetric nature, 5d MSYM theory also suffers from ultraviolet divergences starting at the six-loop level [41] (though there are arguments suggesting that the theory might be rendered finite by some nonperturbative mechanism [42-44]). However, the twisted theory is an exception as one restricts attention to the $Q$-invariant sector: one can make use of the metric independence of the theory to shrink $\Sigma$ or $M$ to a point, thereby reducing the theory to a lower-dimensional one which is renormalizable. Since this process involves a $Q$-exact deformation of the action, it may be thought of as introduction of $Q$-exact regulator terms. In fact, the localization of the path integral we are about to perform is one instance of such a reduction to a lower-dimensional theory by a $Q$-exact deformation. In this case, the twisted theory is reduced to analytically continued Chern-Simons theory.

\subsection{Localization to analytically continued Chern-Simons theory}

We now establish the equivalence between the $\Omega$-deformed twisted 5 d MSYM theory for $\Sigma=D$ and analytically continued Chern-Simons theory. To this end, we view the $5 \mathrm{~d}$ theory on $\Sigma \times M$ as a B-twisted gauge theory on $\Sigma$, regarding $M$ as an internal space whose coordinates are continuous "flavor indices," and interpreting the integration over $M$ in the formula (3.6) etc. as summation over these indices. Then we can localize the path integral for correlation functions just as we did in section 2.4 .

Recall from our discussion in section 2.3 that the boundary condition for our theory is specified by a brane, whose support $\gamma$ is a Lagrangian submanifold of the target space of the chiral multiplet scalar. In the present context, the scalar is the complex gauge field $\mathcal{A}$, and the target space is the space of complex connections on $M$. (If $M$ has a boundary, then

\footnotetext{
${ }^{8}$ The bosonic part of the undeformed action is invariant under phase rotations of $W$. To fix the phase, we need to look at the fermionic part. Alternatively, one can fix it by comparing the Chern-Simons level in our localization formula (3.25) with the identification obtained in [16] from a 6d point of view.
} 
the target space is the space of complex connections obeying a chosen boundary condition.) There is a natural Kähler form on this space, given by

$$
\omega=\int_{M} \operatorname{Tr} \delta A \wedge \star \delta X .
$$

The associated Kähler metric

$$
\mathrm{d} s^{2}=\int_{M} \operatorname{Tr} \delta \mathcal{A} \wedge \star \delta \overline{\mathcal{A}}
$$

is the metric used in the construction of our chiral multiplet action (3.7). So we have equipped the target space with this Kähler form, and $\gamma$ is a Lagrangian submanifold with respect to it. On the boundary, $\mathcal{A}$ is required to be valued in $\gamma$.

We also need to choose the subgroup $H$ which specifies the allowed boundary values of gauge transformations. Previously we chose it to be trivial, that is, we demanded gauge transformations to be trivial on the boundary. This time, we allow all possible gauge transformations that preserve the boundary condition $A_{n}=0$. For the reason explained already, they must be moreover topologically trivial along $M$ on the boundary. Thus, $H$ in the present case is the group of topologically trivial $G$-gauge transformations on $M$, which we denote by $\mathcal{G}$; the corresponding moment map is

$$
\mu=D^{m} X_{m}
$$

Accordingly, $\gamma$ is required to be invariant under the action of $\mathcal{G}$.

In fact, for the purpose of connecting our $5 \mathrm{~d}$ theory to analytically continued ChernSimons theory, we need a stronger condition on $\gamma$ : we require

$$
\gamma=\Gamma \cap \mu^{-1}(0)
$$

for some $\mathcal{G}_{\mathbb{C}}$-invariant submanifold $\Gamma$ of the space of complex connections on $M$, where $\mathcal{G}_{\mathbb{C}}$ is the complexification of $\mathcal{G}$. (As it will become clear, $\Gamma$ is identified with an integration contour for the path integral in the Chern-Simons theory; since this theory has invariance under complex gauge transformations, $\Gamma$ should be invariant under $\mathcal{G}_{\mathbb{C}}$, not just $\mathcal{G}$.) Due to the Kähler form (3.13) being only invariant under $\mathcal{G}$ and not $\mathcal{G}_{\mathbb{C}}$, generically $\Gamma$ itself cannot be a Lagrangian submanifold. However, its restriction $\gamma$ to the $\mathcal{G}$-invariant submanifold $\mu^{-1}(0)$ can be so, and used as the support of our brane. The above form of $\gamma$ is compatible with our localization condition, which actually enforces the restriction $\mu=0$.

The localization procedure is essentially the same as before. We deform the action as

$$
S \rightarrow t^{3} S_{V}+t S_{C}+S_{W}-\frac{1}{2} \delta \int_{D \times M} \sqrt{h} \mathrm{~d}^{5} x \operatorname{Tr}\left(t^{3} \alpha^{\mu \nu} \mathbf{D}_{\mu \nu}^{\prime}+t s \mathbf{F}^{\mu \nu m} \bar{\mu}_{\mu \nu m}\right),
$$

rescale $\bar{\mu}$ as $\bar{\mu} \rightarrow s^{-1} \bar{\mu}$, and send $s \rightarrow \infty$. Then we integrate out the auxiliary fields, whereby we get $\mathbf{F}=0$ and the constraint (2.19) on $\mu$. After that, we equip $D$ with a metric with positive curvature and take $t$ to be very large to find that the path integral localizes to the locus given by the equations

$$
F_{\mu \nu}=\sigma_{\mu}=F_{\mu m}=D_{\mu} X_{m}=0
$$


With $A_{\mu}$ totally gauged away, the equations become

$$
A_{\mu}=\sigma_{\mu}=\partial_{\mu} A_{m}=\partial_{\mu} X_{m}=0
$$

These equations say that the nontrivial part of a localization configuration is specified by the complex gauge field $\mathcal{A}$ which must be constant on $D$. The brane boundary condition requires $\mathcal{A} \in \Gamma \cap \mu^{-1}(0)$, and the constraint coming from $\mathbf{D}^{\prime}$ demands $\mu=0$, which is compatible with the boundary condition. The localization locus is therefore $\Gamma \cap \mu^{-1}(0)$.

Having identified the localization configurations, we integrate over fluctuations around these configurations and over the fermions. For the gauge-fixing condition, we again use the standard one $\hat{h}^{M N} \nabla_{M} A_{N}=0$, where $\hat{h}$ can be any metric on $D \times M$. For us, it is convenient to use $\hat{h}=h_{D} \oplus t^{3 / 2} h_{M}$, for which the corresponding gauge-fixing term is

$$
S_{G}=t^{3} \int_{D \times M} \sqrt{h} \mathrm{~d}^{5} x \operatorname{Tr}\left(\bar{c} \nabla^{\mu} D_{\mu} c+t^{-3 / 2} \bar{c} \nabla^{m} D_{m} c+\left(\nabla^{\mu} A_{\mu}+t^{-3 / 2} \nabla^{m} A_{m}\right)^{2}\right) .
$$

After adding this term to the action, we rescale the fluctuations and the fermions appropriately. The way we do this is slightly different from the rescaling (2.23) considered before, since this time the ghosts have zero modes; the zero-mode parts $c_{0}, \bar{c}_{0}$ of $c, \bar{c}$ are simply constants on $D$, and may be regarded as adjoint-valued scalar fields on $M$. (Recall that we are allowing all gauge transformations that are compatible with the boundary condition $A_{n}=0$.) Writing $c=c_{0}+\tilde{c}, \bar{c}=\bar{c}_{0}+\tilde{\bar{c}}$, we rescale the ghosts as

$$
\left(c_{0}, \tilde{c}, \bar{c}_{0}, \tilde{\bar{c}}\right) \rightarrow\left(t^{-3 / 4} c_{0}, t^{-3 / 2} \tilde{c}, t^{-3 / 4} \bar{c}_{0}, t^{-3 / 2} \tilde{\bar{c}}\right) .
$$

The remaining fields are rescaled as before. Noting that $c$ satisfies the boundary condition $\partial_{n} c=0$ just as gauge transformation parameters do, we then find that to leading order, the fluctuations and the fermion nonzero modes enter the action only through terms that do not depend on the background. Hence, integration over them just produces a constant independent of the background.

The final expression of the localized path integral is similar to the formula (2.27). Unlike the previous case, however, it involves integration over the zero modes $c_{0}, \bar{c}_{0}$. Another difference is that $S_{G}$ contains terms that depend on the background and $c_{0}, \bar{c}_{0}$ :

$$
S_{G 0}=\int_{D} \sqrt{h_{D}} \mathrm{~d}^{2} x \int_{M} \sqrt{h_{M}} \mathrm{~d}^{3} x\left(\bar{c}_{0} \nabla^{m} D_{m} c_{0}+\left(\nabla^{m} A_{m 0}\right)^{2}\right) .
$$

Taking these differences into account, we obtain the localization formula

$$
\langle\mathcal{O}\rangle=\int_{\Gamma \cap \mu^{-1}(0)} \mathcal{D} \mathcal{A}_{0} \mathcal{D} c_{0} \mathcal{D} \bar{c}_{0} \exp \left(S_{0}-S_{G 0}\right) \mathcal{O},
$$

with

$$
S_{0}=\frac{\pi i}{2 e^{2} \varepsilon} \int_{M} \operatorname{Tr}\left(\mathcal{A}_{0} \wedge \mathrm{d} \mathcal{A}_{0}-\frac{2 i}{3} \mathcal{A}_{0} \wedge \mathcal{A}_{0} \wedge \mathcal{A}_{0}\right) .
$$

The piece $S_{G 0}$ in the action that appears in the above formula may be interpreted as a gauge-fixing term for the $3 \mathrm{~d}$ gauge symmetry. So we can drop this piece if we perform the path integral over $\left(\Gamma \cap \mu^{-1}(0)\right) / \mathcal{G}$. 
On the other hand, the restriction of the path integral to the subspace $\mu^{-1}(0)$ amounts to gauge fixing of the noncompact part of the complexified gauge symmetry. The reason is that the equation $\mu=D^{m} X_{m}=0$ is invariant under $\mathcal{G}$ but not under $\mathcal{G}_{\mathbb{C}}$, and using $\mathcal{G}_{\mathbb{C}}$, a generic complex connection can be transformed to one that fixes $\mu=0$. This is actually a familiar fact about the moduli space of vacua in supersymmetric gauge theory: the moduli space is the zero locus of the D- and F-term potentials modulo gauge transformations, but the same space can also be obtained by dropping the D-term equation and taking the quotient with respect to the action of the complexified gauge group. Thus, we can drop the constraint $\mu=0$ and complexify the gauge symmetry, replacing the integration contour with the submanfiold $\Gamma / \mathcal{G}_{\mathbb{C}}$ of the moduli space $\mathcal{M}$ of complex connections on $M$. This mechanism of complexification of the gauge symmetry has been observed previously for $5 \mathrm{~d}$ MSYM theory on $S^{2} \times M[8,10]$.

The above formula can then be rewritten as

$$
\langle\mathcal{O}\rangle=\int_{\Gamma / \mathcal{G}_{\mathbb{C}}} \mathcal{D} \mathcal{A} \exp \left(i k S_{\mathrm{CS}}\right) \mathcal{O},
$$

where

$$
S_{\mathrm{CS}}=\frac{1}{4 \pi} \int_{M} \operatorname{Tr}\left(\mathcal{A} \wedge \mathrm{d} \mathcal{A}-\frac{2 i}{3} \mathcal{A} \wedge \mathcal{A} \wedge \mathcal{A}\right), \quad k=\frac{2 \pi^{2}}{e^{2} \varepsilon}
$$

This is the path integral for Chern-Simons theory at level $k$, with the gauge field analytically continued to a complex connection. Therefore, we have reduced the path integral for the $\Omega$-deformed twisted 5d MSYM theory on $D \times M$ to that for analytically continued ChernSimons theory on $M$, establishing the equivalence between the two theories.

In the Chern-Simons theory, one must specify a convergent middle-dimensional integration cycle in $\mathcal{M}$. In our localization formula, the integration contour $\Gamma / \mathcal{G}_{\mathbb{C}}$ is a Lagrangian submanifold of $\mathcal{M} .^{9}$ A basic example of such a contour is the real contour, represented

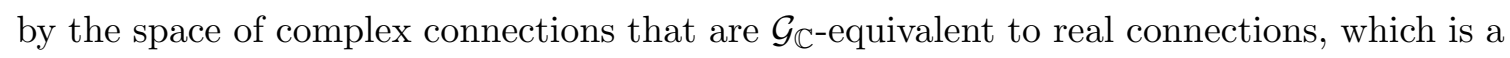
good contour when the Chern-Simons level is real.

\section{3d-3d correspondence}

To conclude our discussion, in the final section we interpret the results we obtained about the $\Omega$-deformed twisted $5 \mathrm{~d}$ MSYM theory from the point of view of the $3 \mathrm{~d}-3 \mathrm{~d}$ correspondence. This allows us to establish the correspondence between the $3 \mathrm{~d} \mathcal{N}=2$ superconformal theory $T[M]$ and analytically continued Chern-Simons theory on $M$. Furthermore, we will see that our construction of the $5 \mathrm{~d}$ theory, together with the $3 \mathrm{~d}-3 \mathrm{~d}$ correspondence, implies a mirror symmetry between $\Omega$-deformed $2 \mathrm{~d}$ theories.

\footnotetext{
${ }^{9}$ The Kähler form on $\mathcal{M}$ is inherited from the space of complex connections: under the identification $\mathcal{M} \simeq \mu^{-1}(0) / \mathcal{G}$, it is represented by the restriction of the $\mathcal{G}$-invariant two-form (3.13) to $\mu^{-1}(0)$. It vanishes on $\Gamma / \mathcal{G}_{\mathbb{C}} \simeq\left(\Gamma \cap \mu^{-1}(0)\right) / \mathcal{G}$ since $\gamma=\Gamma \cap \mu^{-1}(0)$ is a Lagrangian submanifold by assumption. Being an integration cycle of the Chern-Simons theory, $\Gamma / \mathcal{G}_{\mathbb{C}}$ is moreover middle-dimensional in $\mathcal{M}$.
} 


\section{1 $T[M]$ and analytically continued Chern-Simons theory}

Consider the $(2,0)$ theory on $S^{1} \times_{V} \Sigma \times M$, with $S^{1}$ a circle of radius $R$ and $V$ a Killing vector field on $\Sigma$. Here, the space $S^{1} \times_{V} \Sigma$ is a nontrivial $\Sigma$-fibration over $S^{1}$, constructed from the trivial fibration $[0,2 \pi R] \times \Sigma$, by gluing the two ends of the interval $[0,2 \pi R]$ with an action of the isometry $\exp (2 \pi R V)$ on the fiber $\Sigma$. The structure group of the spinor bundle of this space is reduced to $\operatorname{Spin}(2)_{\Sigma} \times \operatorname{Spin}(3)_{M}$, and the R-symmetry group of the theory is $\operatorname{Spin}(5)_{R}$. This is just like the case of $5 \mathrm{~d}$ MSYM theory on $\Sigma \times M$. Thus, we can consider topological twisting analogous to the one applied to that theory.

It is well known that for flat spacetime, the $(2,0)$ theory compactified on $S^{1}$ is equivalent, at low energies, to $5 \mathrm{~d}$ MSYM theory with gauge coupling $e^{2}=4 \pi^{2} R$. In view of this relation, we propose that at energies much smaller than $1 / R$, the above twisted $(2,0)$ theory on $S^{1} \times_{V} \Sigma \times M$ is equivalent to the $\Omega$-deformed twisted 5d MSYM theory on $\Sigma \times M$ constructed in the previous section, with the same gauge coupling and the $\Omega$-deformation given by a Killing vector field proportional to $V$.

Another regime that is relevant to us is the one in which energies are much smaller than $1 / L$, where $L$ is the length scale of $M$. In this regime, the $(2,0)$ theory compactified on $M$ gives $T[M]$ by definition. Hence, the twisted $(2,0)$ theory reduces to a topologically twisted version of $T[M]$ on $S^{1} \times_{V} \Sigma$.

Based on our proposal and this observation, we can show that the $\Omega$-deformed twisted $5 \mathrm{~d}$ MSYM theory is equivalent to the twisted $T[M]$. The argument goes as follows.

We fix an energy scale $E$, and consider the twisted $(2,0)$ theory on $S^{1} \times_{V} \Sigma \times M$ with $R, L \ll 1 / E$. This theory can be described either as the $\Omega$-deformed twisted 5 d MSYM theory on $\Sigma \times M$, with $e^{2}$ and $M$ small, or as the twisted $T[M]$ on $S^{1} \times_{V} \Sigma$, with the $S^{1}$ small. The $5 \mathrm{~d}$ theory is topological on $M$, so we can scale up $M$ if we wish. Likewise, the $3 \mathrm{~d}$ theory is independent of $R$ and we can set it to any value as long as we keep unchanged the isometry $\exp (2 \pi R V$ ) (and other possible fugacity parameters associated to boundaries in $M$ ), for correlation functions on $S^{1} \times_{V} \Sigma$ are supersymmetric indices. (See e.g. [16] for more discussions on this point.)

The last statement suggets that the $5 \mathrm{~d}$ theory depends on $e^{2}$ only through the combination $e^{2} V$, and this is indeed true. To see this, we consider a $Q$-exact deformation of the action similar to the one used in the derivation of the localization formula for $\Sigma=D$ in section 3.2. After such a $Q$-exact deformation, only $S_{V}, S_{C}$ and the boundary term in $S_{W}$ are relevant for the computation of the path integral. The claim then follows from the fact that the dependence on $e^{2}$ coming from the first two is $Q$-exact, while the boundary term of the action depends on $e^{2}$ through the factor $1 / e^{2} \varepsilon$. Thus, we can rescale $e^{2}$ to any value, if we simultaneously rescale $V$ to keep $e^{2} V$ fixed.

Since the $5 \mathrm{~d}$ and $3 \mathrm{~d}$ theories are different descriptions of the same $6 \mathrm{~d}$ theory, they are equivalent, and this is valid at any energy scale $E$, for any values of $e^{2}$ and $R$, and for any metric on $M$. Therefore, we conclude that the $\Omega$-deformed twisted $5 \mathrm{~d}$ MSYM theory on $\Sigma \times M$ is equivalent to the twisted $T[M]$ on $S^{1} \times_{V} \Sigma$. Our argument is depicted in figure 1 .

Now we take $\Sigma=D$. In this case we have shown that the $\Omega$-deformed twisted $5 \mathrm{~d}$ MSYM theory is equivalent to analytically continued Chern-Simons theory. Combined 


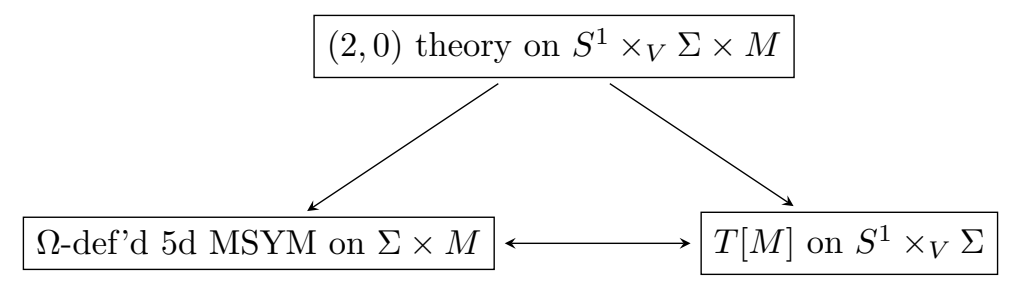

Figure 1. Equivalence between the $\Omega$-deformed twisted 5d MSYM theory and the twisted $T[M]$.

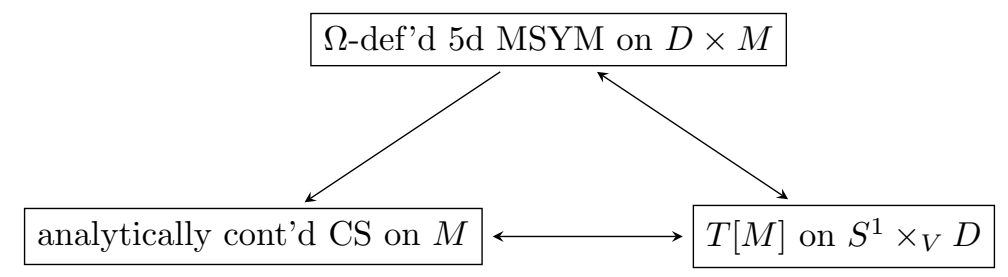

Figure 2. Correspondence between $T[M]$ and analytically continued Chern-Simons theory.

with the equivalence just discussed, this establishes the correspondence between $T[M]$ and the latter theory (figure 2).

Let us briefly comment on an alternative explanation for this correspondence, proposed by Beem et al. [16]. Their approach starts with the same $6 \mathrm{~d}$ setup as ours, namely the $(2,0)$ theory on $S^{1} \times_{V} D \times M$. The main difference is that in their case, in addition to reduction on the $S^{1}$, one considers deforming $D$ to a cigar shape and reducing the theory on the circle fibers of $D$. After doing so, one has a twisted $\mathcal{N}=4$ super Yang-Mills theory on the product of an interval and $M$. Then one can invoke an argument given in [20, 21] and show that the system is equivalent to the Chern-Simons theory. Our derivation has the advantage that it avoids questions concerning the singular point of the geometry, that is the tip of the cigar, where the circle fiber shrinks to a point and the analysis becomes difficult.

In deriving the correspondence between $T[M]$ and analytically continued Chern-Simons theory, we set $\Sigma=D$ and impose boundary conditions of a specific type. Similar localization computations may be carried out for other choices of $\Sigma$ and boundary conditions, and may lead to yet unknown correspondences.

\section{$4.2 \Omega$-deformed mirror symmetry}

The equivalence between the $\Omega$-deformed twisted $5 \mathrm{~d}$ MSYM theory and the twisted $T[M]$ implies more than just the correspondence discussed above. We can use it to find another interesting correspondence which relates two $\Omega$-deformed $2 \mathrm{~d}$ theories.

Consider 5d MSYM theory, compactified and topologically twisted on $M$. In the limit where $M$ is very small, it becomes an $\mathcal{N}=(2,2)$ theory $\widetilde{T}[M]$ in two dimensions. An analysis along the lines of [45] shows that $\widetilde{T}[M]$ is a Landau-Ginzburg model whose target space is the moduli space $\mathcal{M}_{\text {flat }}$ of complex flat connections on $M$, assuming that the flat connections are irreducible. ${ }^{10}$

\footnotetext{
${ }^{10}$ In general, the Landau-Ginzburg model description breaks down at reducible flat connections due to appearance of extra massless modes on $M$ coming from $A_{\mu}, \sigma_{\mu}$ and their superpartners. This echoes the
} 


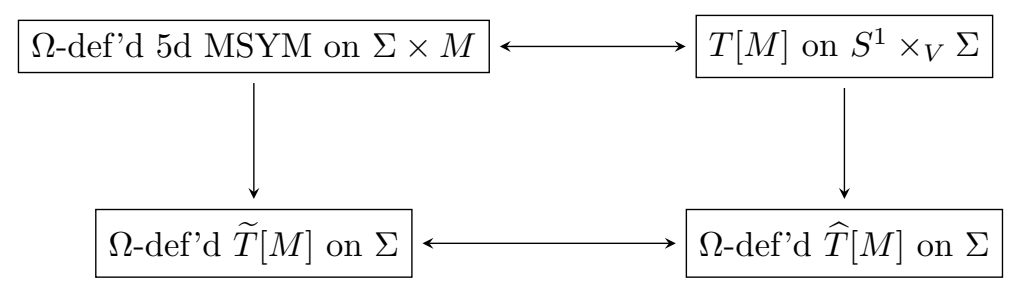

Figure 3. $\Omega$-deformed mirror symmetry.

If we instead start from the $\Omega$-deformed twisted $5 \mathrm{~d}$ MSYM theory on $\Sigma \times M$, then we obtain an $\Omega$-deformed, twisted version of $\widetilde{T}[M]$ on $\Sigma$. The model is more precisely B-twisted, as our construction of the $5 \mathrm{~d}$ theory is based on a B-twisted gauge theory, and the chiral multiplets of the model simply come from their counterparts in the $5 \mathrm{~d}$ theory, containing $\mathcal{A}_{m}$. Alternatively, one may note that generically $\mathrm{U}(1)_{V}$ would be broken by the superpotential, so the twisting should be done with $\mathrm{U}(1)_{A}$. (If the model happens to have a quasi-homogeneous superpotential, one can deform the $5 \mathrm{~d}$ theory so that nonhomogenous terms are generated; then one knows that the $2 \mathrm{~d}$ theory is B-twisted, as the twisting does not change under such a deformation.)

On the other hand, $T[M]$ compactified on $S^{1}$ reduces to an $\mathcal{N}=(2,2)$ theory $\widehat{T}[M]$ in the limit $R \rightarrow 0$. So if we instead start with the twisted version of $T[M]$ formulated on $S^{1} \times_{V} \Sigma$, then we get an $\Omega$-deformed twisted $\widehat{T}[M]$ on $\Sigma$.

Now, combining the facts that (1) the $\Omega$-deformed twisted 5d MYSM theory is topological on $M$; (2) the twisted $T[M]$ on $S^{1} \times_{V} \Sigma$ is independent of $R$ (as long as $R V$ and other fugacities are fixed); and (3) these two theories are equivalent, we deduce that the $\Omega$-deformed twisted $\widetilde{T}[M]$ is equivalent to the $\Omega$-deformed twisted $\widehat{T}[M]$ (figure 3 ).

This equivalence may be thought of as a mirror symmetry. The reason is that while the twisted 5d MSYM theory reduced on $M$ gives rise to a B-twisted Landau-Ginzburg model, reduction of the twisted $T[M]$ on the $S^{1}$ produces an $A$-twisted gauge theory, if $T[M]$ is realized as gauge theory as in $[1,6]$; in particular, it can flow to an A-twisted sigma model in the infrared. This may be seen from the fact that a scalar in the vector multiplet of the $2 \mathrm{~d}$ theory comes from a component of the $3 \mathrm{~d}$ gauge field, which is neutral under the R-symmetry $\mathrm{U}(1)_{R}$ used in the topological twist of the $3 \mathrm{~d}$ theory. Since the scalar is charged under the axial R-symmetry $\mathrm{U}(1)_{A}$, it follows that $\mathrm{U}(1)_{R}$ becomes the vector R-symmetry $\mathrm{U}(1)_{V}$.

Specializing to the case $\Sigma=D$, we can place the correspondence between $T[M]$ and analytically continued Chern-Simons theory (figure 2) and the one between $\widetilde{T}[M]$ and $\widehat{T}[M]$ (figure 3) in a single diagram (figure 4). The result is an intriguing triangle of correspondences that connects analytically continued Chern-Simons theory, $\widetilde{T}[M]$ and $\widehat{T}[M]$.

Using the relation between $\widetilde{T}[M]$ and analytically continued Chern-Simons theory, we can extract information on the superpotential $\widetilde{W}$ of $\widetilde{T}[M]$ as follows.

Integration cycles for the Chern-Simons theory are described by Morse theory, with the real part of ( $i$ times) the Chern-Simons action $i k S_{\mathrm{CS}}$ taken as the Morse function [14].

observation made in $[11,46]$ that the construction of $T[M]$ proposed in $[6,47]$ really captures only the subsector of the full theory, obtained by truncation to the irreducible connections. 


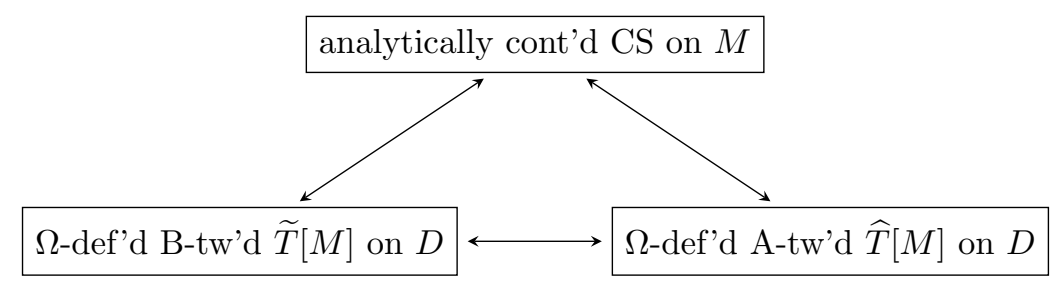

Figure 4. A triangle of correspondences.

To obtain a good integration cycle, one picks a middle-dimensional submanifold $\widetilde{\mathcal{C}}$ of $\mathcal{M}_{\text {flat }}$, and considers downward flow lines that start from some point on $\widetilde{\mathcal{C}}$; let $\mathcal{C}_{\mathcal{A}_{\text {flat }}}$ denote the set of such lines starting from a flat connection $\mathcal{A}_{\text {flat }}$. (If $\mathcal{M}$ has components of different dimensions, $\widetilde{\mathcal{C}}$ is middle-dimensional in each component of fixed dimension.) Then, $\mathcal{C}=$ $\bigcup_{\mathcal{A}_{\text {flat }} \in \widetilde{\mathcal{C}}} \mathcal{C}_{\mathcal{A}_{\text {flat }}}$ represents a desired integration cycle: it is middle-dimensional in the moduli space $\mathcal{M}$ of complex connections, and the path integral is convergent over it since $\operatorname{Re}\left(i k S_{\mathrm{CS}}\right)$ decreases along the flow lines. Given an integration contour $\mathcal{C}$ constructed in this manner, one can compute the partition function by performing the path integral first over $\mathcal{C}_{\mathcal{A}_{\text {flat }}}$, and then over all possible starting points $\mathcal{A}_{\text {flat }}$. The first step defines a function $f$ on $\widetilde{\mathcal{C}}$, with which the partition function can be written as

$$
Z=\int_{\widetilde{\mathcal{C}}} f \mathrm{~d} \mathcal{A}_{\text {flat }},
$$

where $\mathrm{d} \mathcal{A}_{\text {flat }}$ is a holomorphic volume form on $\mathcal{M}_{\text {flat }}$.

For the Chern-Simons theory obtained in our setup, the integration contour $\mathcal{C}$ is represented by the submanifold $\Gamma / \mathcal{G}_{\mathbb{C}}$ of $\mathcal{M}$ which determines the support of the brane in the 5 d theory. This submanifold is Lagrangian, not only middle-dimensional. When $\mathcal{C}$ has this property, $\widetilde{\mathcal{C}}$ is represented by a Lagrangian submanifold of $\mathcal{M}_{\text {flat }}$. Then, $\widetilde{\mathcal{C}}$ naturally defines the support of a brane for $\widetilde{T}[M]$ whose target space is $\mathcal{M}_{\text {flat }}$, and the partition function of $\widetilde{T}[M]$ on $D$ in the presence of this brane is given by $[27]$

$$
Z=\int_{\widetilde{\mathcal{C}}} \mathrm{d} \mathcal{A}_{\text {flat }} \exp \left(\frac{2 \pi i}{\varepsilon} \widetilde{W}\right)
$$

According to the correspondence we found above, this is to be identified with the partition function (4.1) of the Chern-Simons theory. Comparing the two expressions, we see

$$
f=\exp \left(\frac{2 \pi i}{\varepsilon} \widetilde{W}\right)
$$

Hence, information on $\widetilde{W}$ can be extracted by computing the partition function of the Chern-Simons theory over appropriate integration cycles.

\section{Acknowledgments}

We would like to thank Meer Ashwinkumar for helpful discussions. The work of J.Y. is supported by INFN Postdoctoral Fellowship and INFN Research Project ST\&FI. The work of Y.L., M.-C.T. and Q.Z. is supported by NUS Tier 1 FRC Grant R-144-000-316-112. 
Open Access. This article is distributed under the terms of the Creative Commons Attribution License (CC-BY 4.0), which permits any use, distribution and reproduction in any medium, provided the original author(s) and source are credited.

\section{References}

[1] T. Dimofte, S. Gukov and L. Hollands, Vortex counting and Lagrangian 3-manifolds, Lett. Math. Phys. 98 (2011) 225 [arXiv:1006.0977] [INSPIRE].

[2] Y. Terashima and M. Yamazaki, $\mathrm{SL}(2, R)$ Chern-Simons, Liouville and gauge theory on duality walls, JHEP 08 (2011) 135 [arXiv:1103.5748] [INSPIRE].

[3] Y. Terashima and M. Yamazaki, Semiclassical analysis of the $3 d / 3 d$ relation, Phys. Rev. D 88 (2013) 026011 [arXiv:1106.3066] [InSPIRE].

[4] S. Cecotti, C. Cordova and C. Vafa, Braids, walls and mirrors, arXiv:1110.2115 [InSPIRE].

[5] T. Dimofte and S. Gukov, Chern-Simons theory and S-duality, JHEP 05 (2013) 109 [arXiv:1106.4550] [INSPIRE].

[6] T. Dimofte, D. Gaiotto and S. Gukov, Gauge theories labelled by three-manifolds, Commun. Math. Phys. 325 (2014) 367 [arXiv:1108.4389] [INSPIRE].

[7] T. Dimofte, D. Gaiotto and S. Gukov, 3-manifolds and 3d indices, Adv. Theor. Math. Phys. 17 (2013) 975 [arXiv:1112.5179] [InSPIRE].

[8] J. Yagi, 3d TQFT from 6d SCFT, JHEP 08 (2013) 017 [arXiv:1305.0291] [INSPIRE].

[9] S. Lee and M. Yamazaki, 3d Chern-Simons theory from M5-branes, JHEP 12 (2013) 035 [arXiv: 1305.2429] [INSPIRE].

[10] C. Cordova and D.L. Jafferis, Complex Chern-Simons from M5-branes on the squashed three-sphere, arXiv: 1305.2891 [INSPIRE].

[11] T. Dimofte, Complex Chern-Simons theory at level $k$ via the $3 d-3 d$ correspondence, arXiv: 1409.0857 [INSPIRE].

[12] S. Gukov, Three-dimensional quantum gravity, Chern-Simons theory and the A-polynomial, Commun. Math. Phys. 255 (2005) 577 [hep-th/0306165] [INSPIRE].

[13] T. Dimofte, S. Gukov, J. Lenells and D. Zagier, Exact results for perturbative Chern-Simons theory with complex gauge group, Commun. Num. Theor. Phys. 3 (2009) 363 [arXiv:0903.2472] [INSPIRE].

[14] E. Witten, Analytic continuation of Chern-Simons theory, arXiv:1001.2933 [INSPIRE].

[15] S. Pasquetti, Factorisation of $N=2$ theories on the squashed 3-sphere, JHEP 04 (2012) 120 [arXiv:1111.6905] [INSPIRE].

[16] C. Beem, T. Dimofte and S. Pasquetti, Holomorphic blocks in three dimensions, JHEP 12 (2014) 177 [arXiv:1211.1986] [INSPIRE].

[17] M. Taki, Holomorphic blocks for 3d non-Abelian partition functions, arXiv:1303.5915 [INSPIRE].

[18] L.F. Alday, D. Martelli, P. Richmond and J. Sparks, Localization on three-manifolds, JHEP 10 (2013) 095 [arXiv: 1307.6848] [INSPIRE]. 
[19] Y. Imamura, H. Matsuno and D. Yokoyama, Factorization of $S^{3} / Z_{n}$ partition function, Phys. Rev. D 89 (2014) 085003 [arXiv:1311.2371] [InSPIRE].

[20] E. Witten, A new look at the path integral of quantum mechanics, in Surveys in differential geometry. Volume XV. Perspectives in mathematics and physics, Surv. Differ. Geom. 15, Int. Press, Somerville MA U.S.A. (2011), pg. 345 [arXiv: 1009.6032] [INSPIRE].

[21] E. Witten, Fivebranes and knots, Quantum Topol. 3 (2012) 1 [arXiv:1101.3216] [InSPIRE].

[22] L.F. Alday, D. Gaiotto and Y. Tachikawa, Liouville correlation functions from four-dimensional gauge theories, Lett. Math. Phys. 91 (2010) 167 [arXiv:0906.3219] [INSPIRE].

[23] N. Wyllard, $A_{N-1}$ conformal Toda field theory correlation functions from conformal $N=2$ $\mathrm{SU}(N)$ quiver gauge theories, JHEP 11 (2009) 002 [arXiv:0907.2189] [INSPIRE].

[24] T. Kawano and N. Matsumiya, $5 D S Y M$ on $3 D$ sphere and $2 D Y M$, Phys. Lett. B 716 (2012) 450 [arXiv:1206.5966] [inSPIRE].

[25] Y. Fukuda, T. Kawano and N. Matsumiya, 5D SYM and 2D q-deformed YM, Nucl. Phys. B 869 (2013) 493 [arXiv:1210.2855] [InSPIRE].

[26] S. Shadchin, On F-term contribution to effective action, JHEP 08 (2007) 052 [hep-th/0611278] [INSPIRE].

[27] J. Yagi, $\Omega$-deformation and quantization, JHEP 08 (2014) 112 [arXiv:1405.6714] [INSPIRE].

[28] N.A. Nekrasov and S.L. Shatashvili, Quantization of integrable systems and four dimensional gauge theories, in $X V I^{\text {th }}$ International Congress on Mathematical Physics, World Sci. Publ., Hackensack NJ U.S.A. (2010), pg. 265 [arXiv:0908.4052] [INSPIRE].

[29] D. Gaiotto, G.W. Moore and A. Neitzke, Framed BPS states, Adv. Theor. Math. Phys. 17 (2013) 241 [arXiv: 1006.0146] [INSPIRE].

[30] Y. Ito, T. Okuda and M. Taki, Line operators on $S^{1} \times R^{3}$ and quantization of the Hitchin moduli space, JHEP 04 (2012) 010 [arXiv:1111.4221] [INSPIRE].

[31] G.W. Moore, N. Nekrasov and S. Shatashvili, Integrating over Higgs branches, Commun. Math. Phys. 209 (2000) 97 [hep-th/9712241] [INSPIRE].

[32] A. Lossev, N. Nekrasov and S.L. Shatashvili, Testing Seiberg-Witten solution, in Strings, branes and dualities (Cargèse France (1997)), NATO Adv. Sci. Inst. Ser. C Math. Phys. Sci. 520, Kluwer Acad. Publ., Dordrecht The Netherlands (1999), pg. 359 [hep-th/9801061] [INSPIRE].

[33] G.W. Moore, N. Nekrasov and S. Shatashvili, D-particle bound states and generalized instantons, Commun. Math. Phys. 209 (2000) 77 [hep-th/9803265] [InSPIRE].

[34] N.A. Nekrasov, Seiberg-Witten prepotential from instanton counting, Adv. Theor. Math. Phys. 7 (2004) 831 [hep-th/0206161] [INSPIRE].

[35] N. Nekrasov and A. Okounkov, Seiberg-Witten theory and random partitions, in The unity of mathematics, Progr. Math. 244, Birkhäuser, Boston MA (2006), pg. 525 [hep-th/0306238] [INSPIRE].

[36] N. Nekrasov and E. Witten, The omega deformation, branes, integrability and Liouville theory, JHEP 09 (2010) 092 [arXiv: 1002.0888] [INSPIRE].

[37] C. Closset and S. Cremonesi, Comments on $N=(2,2)$ supersymmetry on two-manifolds, JHEP 07 (2014) 075 [arXiv: 1404.2636] [INSPIRE]. 
[38] K. Hori et al., Mirror symmetry, Clay Mathematics Monographs 1, American Mathematical Society, Providence RI U.S.A. (2003) [InSPIRE].

[39] K. Hori and M. Romo, Exact results in two-dimensional $(2,2)$ supersymmetric gauge theories with boundary, arXiv:1308.2438 [INSPIRE].

[40] M. Herbst, K. Hori and D. Page, Phases of $N=2$ theories in $1+1$ dimensions with boundary, arXiv:0803.2045 [INSPIRE].

[41] Z. Bern et al., D = 5 maximally supersymmetric Yang-Mills theory diverges at six loops, Phys. Rev. D 87 (2013) 025018 [arXiv:1210.7709] [INSPIRE].

[42] M.R. Douglas, On D = 5 super Yang-Mills theory and (2,0) theory, JHEP 02 (2011) 011 [arXiv: 1012.2880] [INSPIRE].

[43] N. Lambert, C. Papageorgakis and M. Schmidt-Sommerfeld, M5-branes, D4-branes and quantum 5D super-Yang-Mills, JHEP 01 (2011) 083 [arXiv:1012.2882] [INSPIRE].

[44] N. Lambert, C. Papageorgakis and M. Schmidt-Sommerfeld, Deconstructing $(2,0)$ proposals, Phys. Rev. D 88 (2013) 026007 [arXiv: 1212.3337] [INSPIRE].

[45] M. Bershadsky, A. Johansen, V. Sadov and C. Vafa, Topological reduction of $4 D S Y M$ to $2 D$ o-models, Nucl. Phys. B 448 (1995) 166 [hep-th/9501096] [INSPIRE].

[46] H.-J. Chung, T. Dimofte, S. Gukov and P. Sulkowski, 3d-3d correspondence revisited, arXiv: 1405.3663 [INSPIRE].

[47] T. Dimofte, M. Gabella and A.B. Goncharov, $K$-decompositions and $3 d$ gauge theories, arXiv:1301.0192 [INSPIRE]. 